\title{
EXPLICIT ESTIMATES ON SEVERAL SUMMATORY FUNCTIONS INVOLVING THE MOEBIUS FUNCTION
}

\section{OLIVIER RAMARÉ}

Abstract. We prove that $\left|\sum_{d \leq x} \mu(d) / d\right| \log x \leq 1 / 69$ when $x \geq 96955$ and deduce from that:

$$
\left|\sum_{\left\{\begin{array}{c}
d \leq x, \\
(d, q)=1
\end{array}\right.} \mu(d) / d\right| \log (x / q) \leq \frac{4}{5} q / \varphi(q)
$$

for every $x>q \geq 1$. We also give better constants when $x / q$ is larger. Furthermore we prove that $\left|1-\sum_{d \leq x} \mu(d) \log (x / d) / d\right| \leq \frac{3}{14} / \log x$ and several similar bounds, from which we also prove corresponding bounds when summing the same quantity, but with the additional condition $(d, q)=1$. We prove similar results for $\sum_{d<x} \mu(d) \log ^{2}(x / d) / d$, among which we mention the bound $\left|\sum_{d \leq x} \mu(d) \log ^{2}(x / d) / d-2 \log x+2 \gamma_{0}\right| \leq \frac{5}{24} / \log x$, where $\gamma_{0}$ is the Euler constant. We complete this collection by bounds such as

$$
\left|\sum_{\left\{\begin{array}{c}
d \leq x, \\
(d, q)=1
\end{array}\right.} \mu(d)\right| / x \leq \frac{q}{\varphi(q)} / \log (x / q) .
$$

We also provide all these bounds with variations where $1 / \log x$ is replaced by $1 /(1+\log x)$.

\section{INTRODUCTION}

Explicit estimates in multiplicative number theory have a long history. Concerning prime-related questions, one can distinguish between two main lines of inquiry: estimates on the Chebyshev $\psi$-function and estimates for the summatory function $M$ of the Moebius function. In the first case the explicit formula for the $\psi$-function enables us to introduce in the argument the result of heavy computations regarding the zeros of the Riemann $\zeta$-function; see for instance 22 and 23 . This is so because the residues of the Mellin transform of the $\psi$-function, i.e., $-\zeta^{\prime}(s) / \zeta$, are known: they are simply equal to 1 counted with multiplicity. No such fact happens in the case of the Moebius function, the Mellin transform that appears being $1 / \zeta$. No one has yet obtained an explicit error term for the function $M$ from the Mellin transform / Perron formula machinery, though there are no theoretical obstructions. The implied constants are, however, expected to be too large for any decent use.

Once the analytical path is discarded, two distinct paths of inquiries have been used for the summatory function $M(x)=\sum_{d \leq x} \mu(d)$ : either follow the idea of Chebyshev, (see [13, 66, 8]) or follow an idea of Landau: Landau proved that $\psi(x) \sim x$ is equivalent to $M(x)=o(x)$ and we need a quantitative version of it. See also [12 for these kind of questions. This path is followed in 24] and continued

Received by the editor March 27, 2013 and, in revised form, November 9, 2013.

2010 Mathematics Subject Classification. Primary 11N37, 11Y35; Secondary 11A25.

Key words and phrases. Explicit estimates, Moebius function. 
in [9. Recently in 20, I trode a similar path but relied on a more efficient set of identities.

In practice, one needs estimates for families of functions that are derived from the Moebius function, with as small a loss as one can manage. There are three main variations, and we deal here with two of them; see [21 for the third family. The first problem is to go from $M(x)$ to $m(x)=\sum_{n<x} \mu(d) / d$ and the second one is to add a coprimality condition $(d, q)=1$ for some fixed $q$. Let us introduce some actors before continuing the description of the present work. We define

$$
m_{q}(x)=\sum_{\substack{n \leq x,(n, q)=1}} \mu(n) / n, \quad m(x)=m_{1}(x)
$$

Note that [11, Lemma 10.2] already proposed explicit wide ranging estimates. This investigation is extended in [21] to the second family

$$
\check{m}_{q}(x)=\sum_{\substack{n \leq x,(n, q)=1}} \mu(n) \log (x / n) / n, \quad \check{m}(x)=\check{m}_{1}(x),
$$

and, with some applications in mind, we also cover the family

$$
\check{\check{m}}_{q}(x)=\sum_{\substack{n \leq x,(n, q)=1}} \mu(n) \log ^{2}(x / n) / n, \quad \check{m}(x)=\check{\check{m}}_{1}(x) .
$$

The general problem consists in getting estimates for $m(x)$ and $\check{m}(x)$ from $M(x)=$ $\sum_{d \leq x} \mu(d)$. A summation by parts loses a factor of $\log x$. As kindly pointed out to me by H. Diamond and is clear in [3], a method of A. Axer from [1] already provides an answer to this problem. This method can be found presented in an elementary way in [25, Theorem 2.5], and in [15. Theorem 8.1] and in a more refined setting in [7. Lemma 3.1] (see also [4, Lemma 5.7]).

As an extension of the method of Axer, M. Balazard in [3], furthering work of R.A. MacLeod [14], developed a line of work that led to good identities linking $m(x)$ to $M(x)$ and $\check{m}(x)$ also to $M(x)$. A consequence is the following theorem.

Theorem 1.1 (Balazard). We have, when $x \geq 1$ :

$$
|m(x)| \leq \frac{|M(x)|}{x}+\frac{1}{x^{2}} \int_{1}^{x}|M(t)| d t+\frac{8}{3 x} .
$$

One of the advantages of the method and identity used in 3 is that it implies a differentiable function instead of the fractional-part-function as in Axer's work and this leads to a much smoother treatment.

We readily deduce from the above that

$$
|m(x)| \leq\left(\frac{3}{2}+o(1)\right) \exp \left(-\max _{x^{7 / 8} \leq t \leq x} \log \frac{2+|M(t)|}{t}\right)+\mathcal{O}\left(x^{-1 / 4}\right) .
$$

This is an excellent quantitative link between the error term of $m(x)$ and the one of $M(x)$, though it is not perfect: if we assume that $M(t) \ll t^{3 / 4}$ we deduce from the above that $m(x) \ll x^{-7 / 32}$, while one would like to infer that $m(x) \ll x^{-1 / 4}$. This kind of identity is used in [20, Corollary 1.2], where I relied on an identity due to El Marraki in [10]. By using the new identity above we improve on this work. 
Theorem 1.2. We have

$$
\left|\sum_{n \leq x} \mu(n) / n\right| \leq \frac{0.0144 \log x-0.1}{(\log x)^{2}} \quad(x \geq 463421) .
$$

The following simpler bounds also hold true:

$$
(\log x)\left|\sum_{n \leq x} \mu(n) / n\right| \leq\left\{\begin{array}{l}
1 / 69 \quad \text { when } x \geq 96955, \\
1 / 65 \quad \text { when } x \geq 60298, \\
1 / 40 \quad \text { when } x \geq 24270,
\end{array},\left\{\begin{array}{l}
1 / 25 \quad \text { when } x \geq 3470 \\
1 / 20 \quad \text { when } x \geq 1426 \\
1 / 12 \quad \text { when } x \geq 687
\end{array}\right.\right.
$$

We can also replace $1 / 69$ by 0.0144 .

This result improves by a factor a bit larger than 2 the previous estimate of 20 ] and by a factor a bit more than $31 / 2$ the estimate of [10].

Corollary 1.3.

$$
\left|\sum_{n \leq x} \mu(n) / n\right| \leq \begin{cases}1 & \text { when } x \geq 1 \\ 1 / 10 & \text { when } x \geq 7 \\ 1 / 20 & \text { when } x \geq 41 \\ 1 / 100 & \text { when } x \geq 694\end{cases}
$$

By studying the proof of Theorem 1.1 and another identity by the same author concerning $\check{m}(x)$, I obtained the following result.

Theorem 1.4. We have, when $x \geq 1$ :

$$
|\check{m}(x)-1| \leq \frac{\frac{7}{4}-\gamma_{0}}{x^{2}} \int_{1}^{x}|M(t)| d t+\frac{2}{x} .
$$

Here $\gamma_{0}$ is the Euler constant.

This second very simple inequality (in particular, the term $|M(x)| / x$ does not appear) leads to a much more surprising result. Sections 2 and 3 contain somewhat more precise versions (i.e., identities) and it is in fact these forms that we use (enabling us in practice to reduce the coefficient $\frac{7}{4}-\gamma_{0}=1.172 \cdots$ to 0.321 , see Section 4).

Theorem 1.5. We have

$$
|\check{m}(x)-1| \leq \frac{0.00257 \log x-0.0077}{(\log x)^{2}} \quad(x \geq 3846) .
$$

The following simpler bound also holds:

$$
|\check{m}(x)-1| \log x \leq \begin{cases}0.213<3 / 14 & \text { when } x \geq 1 \\ 0.0203<4 / 197 & \text { when } x \geq 16 \\ 1 / 389 & \text { when } x \geq 3155 .\end{cases}
$$

Corollary 1.6.

$$
\left|-1+\sum_{n \leq x} \mu(n) \log (x / n) / n\right| \leq \begin{cases}1 & \text { when } x \geq 1 \\ 1 / 125 & \text { when } x \geq 7 \\ 1 / 500 & \text { when } x \geq 44 \\ 1 / 1250 & \text { when } x \geq 222\end{cases}
$$


We follow a similar path with $\check{\check{m}}$, the relevant identities being much more cumbersome (see Lemma 5.1). We get the following.

Theorem 1.7. We have, when $x \geq 1$ :

$$
\left|\check{\check{m}}(x)-2 \log x+2 \gamma_{0}\right| \leq 2\left(\gamma_{0}-\frac{1}{2}\right)^{2} \frac{|M(x)|}{x}+\frac{3 / 2}{x^{2}} \int_{1}^{x}|M(t)| d t+\frac{4+2 \gamma_{0}}{x} .
$$

The good surprise is that the term $|M(x)| / x$ is affected with a very small coefficient. Section 5 contains a more precise identity that led to this inequality. Lacking, however, a positivity argument we used in the case of $m(x)$ and $\check{m}(x)$, we cannot proceed as with those: the coefficient $3 / 2$ has to be taken at flat value (well, 1.46 is available).

Theorem 1.8. We have

$$
\left|\check{m}(x)-2 \log x+2 \gamma_{0}\right| \leq \frac{0.00965 \log x-0.0818}{(\log x)^{2}} \quad(x \geq 10) .
$$

The following simpler bounds also hold:

$$
\left|\check{m}(x)-2 \log x+2 \gamma_{0}\right| \log x \leq \begin{cases}0.2062<5 / 24 & \text { when } x>1, \\ 1 / 103 & \text { when } x \geq 9 .\end{cases}
$$

More surprisingly, it numerically seems that the function $\check{m}(x)-2 \log x+2 \gamma_{0}$ is non-increasing. We formulate that formally in the form of a conjecture:

Conjecture. The function $\check{\check{m}}(x)-2 \log x+2 \gamma_{0}$ is positive decreasing.

One would need a representation for this difference that exhibits some positivity. The representation we have contains oscillating terms, typically $M(t)$. Similarly, Section 13 contains an equally surprising question whose veracity is sustained by numerical results. The function $\check{m}(x)-2$ does not exhibit such a behaviour; for instance, it changes its sign of variations around $x=5$.

The coprimality condition is somewhat difficult to handle from a numerical point of view. The classical path (used for instance in [16, near (7)]) consists in determining a function $g_{q}$ such that $\mathbb{1}_{(n, q)=1} \mu(n)=g_{q} \star \mu(n)$, where $\star$ denotes the arithmetic convolution product. The drawback of this method is that the support of $g_{q}$ is not bounded, and indeed, we have

$$
\begin{cases}g_{q}\left(p^{k}\right)=1 & \text { when } p \mid q \text { and } k \geq 0 \\ g_{q}\left(p^{k}\right)=0 & \text { when }(p, q)=1 \text { and } k \geq 1 .\end{cases}
$$

We proposed in [19] an approach via the Liouville function $\lambda(n)$ (the completely multiplicative function that is 1 on integers that have an even number of prime factors, counted with multiplicity, and -1 otherwise). Such an approach splits the evaluation into three steps: expressing $m_{q}(x)$ in terms of $\ell_{q}$, where

$$
\ell_{q}(x)=\sum_{\substack{n \leq x,(n, q)=1}} \lambda(n) / n, \quad \ell(x)=\ell_{1}(x)
$$

then expressing $\ell_{q}(x)$ in terms of $\ell(y)$; and finally expressing $\ell(x)$ in terms of $m(y)$. It turns out that we can combine the first and the third step in a single one, allowing for some non-trivial savings. This time, the drawback of this new method is that the intermediate computations required are heavier and require much more RAM memory than in the previous method (see section 7). Here is our starting lemma. 
Lemma 1.9. We have

$$
m_{q}(x)=\sum_{d \mid q} \frac{\mu^{2}(d)}{d} \sum_{\substack{w \leq \sqrt{x / d}, w \mid q^{\infty}}} m\left(\frac{x}{d w^{2}}\right) / w^{2}
$$

where $w \mid q^{\infty}$ means that every prime factor of $w$ divides $q$. The same identity holds with $\check{m}_{q}$ (resp. $\check{\check{m}}_{q}$ ) and $\check{m}$ (resp. $\check{\check{m}}$ ) instead respectively of $m_{q}$ and $m$.

Proof. We use the decomposition $\mathbb{1}_{(n, q)=1} \mu(n)=g_{q} \star \mu(n)$ where $g_{q}$ is defined in (1.5) and get

$$
m_{q}(x)=\sum_{a \leq x} \frac{g_{q}(a)}{a} m(x / a) .
$$

Next we decompose $a$ in the form $a=d w^{2}$ where $d$ is squarefree. The lemma follows readily.

An alternative proof consists in combining [19, Lemmas 1.1-1.3] and binding the two squares in a single variable $w$. This identity has an almost immediate consequence.

Corollary 1.10. For any real number $x \geq 1$ and any positive integer $q$, we have

$$
0 \leq \check{m}_{q}(x)=\sum_{\substack{n \leq x,(n, q)=1}} \mu(n) \log (x / n) / n \leq 1.00303 \cdot q / \varphi(q) .
$$

The constant 1.00303 can be replaced by the optimal one: $\check{m}(30)$. Indeed, Balazard established this for $\check{m}(x)$ in [2] and Lemma 1.9 enables us to extend it. These kinds of wide-ranging estimate is useful. We proceed in the same way with $\check{m}(x)$. We first establish that $0 \leq \check{m}(x) \leq 2 \log x$ by combining numerical verifications and Theorem 1.8, from which we infer the following.

Corollary 1.11. For any real number $x \geq 1$ and any positive integer $q$, we have

$$
0 \leq \check{\check{m}}_{q}(x)=\sum_{\substack{n \leq x,(n, q)=1}} \mu(n) \log ^{2}(x / n) / n \leq 2 \log x \cdot q / \varphi(q) .
$$

In both corollaries, $q$ is not bounded with respect to $x$. The factor $q / \varphi(q)$ is shown to be necessary by selecting $q$ to be the product of every prime not more than $x$. With some more work, this method leads to the following theorem:

Theorem 1.12. We have, when $1 \leq q<x$, where $q$ is an integer and $x$ a real number,

$$
\frac{\varphi(q)}{q} \log (x / q)\left|m_{q}(x)\right| \leq \begin{cases}0.78<4 / 5 & \text { when } x / q>1 \\ 5 / 16 & \text { when } x / q \geq 687 \\ 17 / 125 & \text { when } x / q \geq 24233\end{cases}
$$

In these estimates, we can replace $\varphi(q) / q$ by $1 / \prod_{p \mid q}\left(1+p^{-1}\right)$.

We specify for clarity that one cannot, at least from our proofs, replace $\varphi(q) / q$ by $1 / \prod_{p \mid q}\left(1+p^{-1}\right)$ in Corollaries 1.10 and 1.11. It is sometimes better in usage to have $1+\log x$ instead of $\log x$. 
Theorem 1.13. We have, when $1 \leq q<x$, where $q$ is an integer and $x$ a real number,

$$
\frac{\varphi(q)}{q}(1+\log (x / q))\left|m_{q}(x)\right| \leq \begin{cases}1.694<17 / 10 & \text { when } x / q>1 \\ 1 / 2 & \text { when } x / q \geq 296 \\ 5 / 38 & \text { when } x / q \geq 882 \\ 1 / 7 & \text { when } x / q \geq 11811\end{cases}
$$

In these estimates, we can replace $\varphi(q) / q$ by $1 / \prod_{p \mid q}\left(1+p^{-1}\right)$.

This improves on the corresponding estimates proved in [19. Prior to this paper, the sole estimate on $m_{q}(x)$ seems to be [11, Lemma 10.2] which bounds $\left|m_{q}(x)\right|$ uniformly by 1 . Some numerical investigations produced the example

$$
\left|m_{2}(10)\right|=\frac{31}{105} \geq 0.260 \frac{2}{\log (10 / 2)}
$$

and I have not been able to get any example with a lower bound larger than $0.260 \ldots$ for $\left|m_{q}(x)\right| \frac{\varphi(q)}{q} \log (x / r)$ when $x \geq 3$. It thus seems likely that

$$
\left|m_{q}(x)\right| \stackrel{?}{\leq} \frac{0.261 q}{\varphi(q) \log (x / q)}, \quad(x \geq 3, x>q \geq 1) .
$$

The inequality (9.1) obtained below shows that (1.8) holds true when $x / q \geq 2438$, but this leaves still infinitely many cases to cover.

Theorem 1.14. We have, when $1 \leq q<x$, where $q$ is an integer and $x$ a real number,

$$
\frac{q}{\varphi(q)} \log (x / q)\left|\check{m}_{q}(x)-\check{m}_{q}^{\sharp}(x)\right| \leq \begin{cases}1 / 4 & \text { when } x / q>1, \\ 1 / 5 & \text { when } x / q \geq 100, \\ 0.0538<2 / 37 & \text { when } x / q \geq 3158,\end{cases}
$$

where we use the notation

$$
\check{m}_{q}^{\sharp}(x)=\sum_{\substack{d|q, w| q^{\infty} \\ d w^{2} \leq x}} \frac{\mu^{2}(d)}{d w^{2}} .
$$

In these estimates, we can replace $\varphi(q) / q$ by $1 / \prod_{p \mid q}\left(1+p^{-1}\right)$.

Using the same notations, we get the following variations.

Theorem 1.15. We have, when $1 \leq q<x$, where $q$ is an integer and $x$ a real number,

$$
\frac{q}{\varphi(q)}(1+\log (x / q))\left|\check{m}_{q}(x)-\check{m}_{q}^{\sharp}(x)\right| \leq \begin{cases}1 & \text { when } x / q>1, \\ 1 / 5 & \text { when } x / q \geq 171, \\ 0.066<1 / 15 & \text { when } x / q \geq 3150 .\end{cases}
$$

At this level of generality, it is not possible to simplify the main term $\check{m}_{q}^{\sharp}(x)$. We proceed in a similar fashion for $\check{\check{m}}_{q}$ 
Theorem 1.16. We have, when $1 \leq q<x$, where $q$ is an integer and $x$ a real number,

$$
\frac{\varphi(q)}{q} \log (x / q)\left|\check{\check{m}}_{q}(x)-\check{m}_{q}^{\sharp}(x)\right| \leq \begin{cases}4 / 19 & \text { when } x / q>1, \\ 1 / 48 & \text { when } x / q \geq 5 .\end{cases}
$$

We have used the notation

$$
\check{\check{m}}_{q}^{\sharp}(x)=2 \sum_{\substack{d|q, w| q^{\infty} \\ d w^{2} \leq x}} \frac{\mu^{2}(d)\left(\gamma_{0}-\log \left(x /\left(d w^{2}\right)\right)\right.}{d w^{2}} .
$$

In these estimates, we can replace $\varphi(q) / q$ by $1 / \prod_{p \mid q}\left(1+p^{-1}\right)$.

Theorem 1.17. We have, when $1 \leq q<x$, where $q$ is an integer and $x$ a real number,

$$
\frac{\varphi(q)}{q}(1+\log (x / q))\left|\check{\check{m}}_{q}(x)-\check{m}_{q}^{\sharp}(x)\right| \leq \begin{cases}1.155<7 / 6 & \text { when } x / q>1, \\ 3 / 17 & \text { when } x / q \geq 2, \\ 1 / 22 & \text { when } x \geq 6 .\end{cases}
$$

In these estimates, we can replace $\varphi(q) / q$ by $1 / \prod_{p \mid q}\left(1+p^{-1}\right)$.

We finally complete this series of results with one concerning $M_{q}(x)$, where we follow the previous convention:

$$
M_{q}(x)=\sum_{\substack{n \leq x,(n, q)=1}} \mu(n), \quad M(x)=M_{1}(x) .
$$

We adapt Lemma 1.9 in (16.1) and a similar routine leads to the following.

Theorem 1.18. We have, when $1 \leq q<x$, where $q$ is an integer and $x$ a real number,

$$
\frac{\varphi(q)}{q} \log (x / q)\left|M_{q}(x)\right| / x \leq \begin{cases}0.997 & \text { when } x / q>1 \\ 0.429<9 / 20 & \text { when } x / q \geq 490 \\ 1 / 5 & \text { when } x / q \geq 4536 \\ 0.0918<9 / 98 & \text { when } x / q \geq 48513\end{cases}
$$

In these estimates, we can replace $\varphi(q) / q$ by $1 / \prod_{p \mid q}\left(1+p^{-1}\right)$.

Theorem 1.19. We have, when $1 \leq q<x$, where $q$ is an integer and $x$ a real number,

$$
\frac{\varphi(q)}{q}(1+\log (x / q))\left|M_{q}(x)\right| / x \leq \begin{cases}1 & \text { when } x / q>1 \\ 1 / 2 & \text { when } x / q \geq 490 \\ 1 / 5 & \text { when } x / q \geq 7100 \\ 39 / 400 & \text { when } x / q \geq 48645\end{cases}
$$

In these estimates, we can replace $\varphi(q) / q$ by $1 / \prod_{p \mid q}\left(1+p^{-1}\right)$. 
Scripts. All the computations used have been achieved via GP/PARI (see [18]) often sped up by using gp2c as described for instance in [5]. To give a flavor, we have used for instance the command gp-run -g AsymptoticBoundsFor_M.gp. The flag "-g" enables automatic memory management and garbage collection. The computations have been run on a 64-bit dual core running at $3.0 \mathrm{GHz}$ and having 8 Gbytes of RAM. One also wants to increase the heap size via allocatemem(7500000000). The amount of RAM available was decided on all the ranges of initial computation; we did not rely on any swapping capacity, as this slows dramatically the computations. Practically we increased the range of the bound under inspection until most of the RAM was used. I am indebted to Bill Alombert for helping me in writing many scripts in a near optimal way. The important scripts are available online at

http://math. univ-lille1.fr ramare/index.html\#GP

\section{An identity for $m(x)$, Proof of Theorem 1.2 And of Lemma 1.3}

Here is the main identity we use, taken from [2, Proof of proposition 6]:

$$
m(x)=\frac{M(x)}{x}+\frac{4\left(1-x^{-1}\right)^{2}}{x}-\frac{4\left(1-x^{-1}\right)^{3}}{3 x^{2}}+\frac{1}{x} \int_{1}^{x} M(x / t) \varepsilon_{1}^{\prime}(t) d t
$$

where

$$
\varepsilon_{1}^{\prime}(t)=\left(\frac{(2\{t\}-1) t+\{t\}-\{t\}^{2}}{t^{2}}\right)^{2}
$$

is the derivative at every non-integer point of

$$
\varepsilon_{1}(t)=\frac{1}{3}-\frac{1}{3 t}+\frac{4}{3} \frac{\{t\}^{3}-\frac{3}{2}\{t\}^{2}+\frac{1}{2}\{t\}}{t^{2}}-\frac{1}{3} \frac{\{t\}^{4}-2\{t\}^{3}+\{t\}^{2}}{t^{4}} .
$$

Moreover, $0 \leq \varepsilon_{1}^{\prime}(t) \leq 1 / t^{2}$. Let us recall [20, Theorem 1.1]

Lemma 2.1. For $D \geq 1078853$, we have

$$
\left|\sum_{d \leq D} \mu(d)\right| \leq \frac{0.0130 \log D-0.118}{(\log D)^{2}} D .
$$

We use the following computations.

Lemma 2.2. We have, with $D_{0}=1078853$

$$
\int_{\substack{1 \leq t \leq D_{0}, M(t) \geq 0}} M(t) d t=58909800, \quad \int_{\substack{1 \leq t \leq D_{0}, M(t) \leq 0}} M(t) d t=-54647032 .
$$

We use the file CompIntM.gp whose main function is getintM. Since we have this script at hand, we also prove the following version that will be of use in Section 4 .

Lemma 2.3. We have, with $D_{0}=464402$,

$$
\int_{\substack{1 \leq t \leq D_{0}, M(t) \geq 0}} M(t) d t=15512101, \quad \int_{\substack{1 \leq t \leq D_{0}, M(t) \leq 0}} M(t) d t=-14504264 .
$$

Lemma 2.4. The function

$$
x \mapsto \frac{\log x}{3} \int_{x D_{0} /\left(x+D_{0}\right)}^{x / 2} \frac{0.0130 \log u-0.118}{(\log u)^{2}} \frac{u d u}{(x-u)^{2}}+\frac{58909800 \log x}{x^{2}}
$$

is increasing to $\frac{1}{3} 0.0130(1-\log 2)=0.00132 \cdots$. As a conclusion, it is $\leq 0.00134$ when $1078853=D_{0} \leq x$. 
Proof. Let us denote by $x \mapsto f(x) \log x$ the function to be studied. In practice, it would be enough to plot the function and check that it is "numerically" increasing. Here we can show that it is indeed increasing and as a matter of fact, the function $x \mapsto f(x)$ is increasing. We recall that

$$
\frac{d}{d x} \int_{a}^{h(x)} H(x, u) d x=\int_{a}^{h(x)} \frac{\partial}{\partial x} H(x, u) d u+h^{\prime}(X) H(x, h(x))
$$

provided that the integrals $\int_{a}^{h(x)}\left|\frac{\partial}{\partial x} H(x, u)\right| d u$ and $\int_{a}^{h(x)}|H(x, u)| d u$ are uniformly bounded in a neighborhood of the point where we take the derivative. Here, this formula yields

$$
\begin{aligned}
f^{\prime}(x) & =\frac{1}{6} \frac{0.0130 \log (x / 2)-0.118}{(\log (x / 2))^{2}} \frac{x}{(x-x / 2)^{2}} \\
+ & \frac{D_{0}^{2}}{3\left(x+D_{0}\right)^{2}} \frac{0.0130 \log \left(x D_{0} /\left(x+D_{0}\right)\right)-0.118}{\left(\log \left(x D_{0} /\left(x+D_{0}\right)\right)\right)^{2}} \frac{x D_{0} /\left(x+D_{0}\right)}{\left(x-x D_{0} /\left(x+D_{0}\right)\right)^{2}} \\
& -\frac{2}{3} \int_{x D_{0} /\left(x+D_{0}\right)}^{x / 2} \frac{0.0130 \log u-0.118}{(\log u)^{2}} \frac{u d u}{(x-u)^{3}}-\frac{58909800}{x^{3}} .
\end{aligned}
$$

The sum of the two last terms is larger than

$$
-\frac{2}{3} \frac{0.0130 \log x-0.118}{(\log x)^{2}} \frac{(x / 2)^{2}}{x^{3} / 8}-\frac{58909800}{x^{3}} \geq \frac{-0.00682}{x \log x}
$$

provided that $x \geq D_{0}=1078853$. The reader will easily conclude the proof that $f$ is indeed increasing. Regarding the value at infinity, by using equivalents, we deduce that it is

$$
\lim _{x \rightarrow \infty} \frac{\log x}{3} \int_{1}^{x / 2} \frac{0.0130}{\log u} \frac{u d u}{(x-u)^{2}}=\lim _{x \rightarrow \infty} \frac{0.0130}{3} \int_{1}^{x / 2} \frac{u d u}{(x-u)^{2}}
$$

which is also $\frac{0.0130}{3} \int_{0}^{1 / 2} \frac{v d v}{(1-v)^{2}}=0.0130(1-\log 2) / 3$.

To use (2.1), we have to handle the last term. We note that, when $n$ is an integer, we have

$$
\begin{aligned}
\int_{n}^{n+1} & |M(x / t)| \varepsilon_{1}^{\prime}(t) d t=\max _{n \leq t \leq n+1}|M(x / t)|\left(\varepsilon_{1}(n+1)-\varepsilon_{1}(n)\right) \\
\quad= & \max _{n \leq t \leq n+1}|M(x / t)|\left(\frac{1}{3 n}-\frac{1}{3(n+1)}\right)=\frac{1}{3} \int_{n}^{n+1} \max _{n \leq t \leq n+1}|M(x / t)| d t / t^{2} .
\end{aligned}
$$

Thus

$$
\begin{aligned}
\int_{1}^{x}|M(x / t)| \varepsilon_{1}^{\prime}(t) d t \leq \int_{1}^{x / D_{0}}|M(x / t)| \varepsilon_{1}^{\prime}(t) d t+\frac{58909800}{x} & \\
\leq & \frac{x}{3} \int_{1}^{x / D_{0}} \frac{0.0146 \log (x /(t+1))-0.1098}{(\log (x /(t+1)))^{2}} \frac{d t}{t^{2}(t+1)}+\frac{58909800}{x} \\
\leq & \frac{x}{3} \int_{x D_{0} /\left(x+D_{0}\right)}^{x / 2} \frac{0.0146 \log u-0.1098}{(\log u)^{2}} \frac{u d u}{(x-u)^{2}}+\frac{58909800}{x} \\
& \leq 0.00134 x / \log x
\end{aligned}
$$


by Lemma 2.4, This yields

$$
|m(x)| \leq \frac{0.0130 \log x-0.118}{(\log x)^{2}}+\frac{4}{x}+\frac{0.00134}{\log x} \leq \frac{0.0144 \log x-0.118}{(\log x)^{2}}
$$

when $x \geq D_{0}$. We checked it numerically for $619000 \leq x \leq 3 \cdot 10^{7}$. Theorem 1.2 and Corollary 1.3 follow readily. The script used is AsymptoticBoundsFor.m.gp whose main function is getboundsbis. The function modelmult has to be chosen based on need.

\section{An IDENTITy For $\check{m}(x)$ AND PRoof of Theorem 1.4}

As a preparation for the next section, we prove more than is strictly required here (only case $\ell=0$ of what follows is needed in this section). A simple use of the Euler-MacLaurin summation formula gives, when $\ell$ is a non-negative integer that

$$
\begin{aligned}
H_{\ell}(x) & =\sum_{n \leq x} \frac{\log ^{\ell} n}{n} \\
& =\frac{(\log x)^{\ell+1}}{\ell+1}+\gamma_{\ell}-B_{1}(x) f_{\ell}(x)+\frac{B_{2}(x)}{2} f_{\ell}^{\prime}(x)+\varepsilon_{6, \ell}(x)
\end{aligned}
$$

with $f_{\ell}(t)=(\log t)^{\ell} / t$ and the classical notation $B_{1}(t)=\{t\}-\frac{1}{2}, b_{2}(x)=x^{2}-x+\frac{1}{6}$, $B_{2}(t)=b_{2}(\{t\})$ as well as

$$
\varepsilon_{6, \ell}(x)=\frac{1}{2} \int_{x}^{\infty} B_{2}(t) f_{\ell}^{\prime \prime}(t) d t .
$$

The constant $\gamma_{\ell}$ is the Euler-Stieltjes constant, and $\gamma_{0}$ is the classical Euler constant, while $\gamma_{1}=-0.07281584548 \cdots$.

Lemma 3.1. When $m$ is a non-negative integer and $y \geq 1$ a real number we have

$$
\int_{1}^{y} t \log ^{m} t d t / m !=\frac{y^{2}}{2} \sum_{\substack{a+b=m, a, b \geq 0}} \frac{(-1)^{b}(\log y)^{a}}{a ! 2^{b}}+\frac{(-1)^{m+1}}{2^{m+1}} .
$$

Proof. The proof goes by generating series. We consider

$$
\begin{aligned}
\sum_{n \geq 0} \frac{z^{n}}{n !} \int_{1}^{y} t \log ^{n} t d t & =\int_{1}^{y} t^{1+z} d t=\frac{y^{2} y^{z}-1}{2+z} \\
& =\frac{y^{2}}{2} \sum_{a \geq 0} \frac{z^{a}(\log y)^{a}}{a !} \sum_{b \geq 0}(-z / 2)^{b}-\frac{1}{2} \sum_{b \geq 0}(-z / 2)^{b} .
\end{aligned}
$$

Identification leads to the lemma.

Furthermore, still following Balazard's notation from [3] and [2] in case $\ell=0$,

$$
\begin{aligned}
\beta_{2, \ell}(x) & =\frac{2}{x} \int_{0}^{x} \sum_{n \leq t} \frac{t}{n} \log ^{\ell}(t / n) d t \\
& =\frac{2}{x} \sum_{n \leq x} n \int_{1}^{x / n} u \log ^{\ell} u d u \\
& =\sum_{n \leq x}\left(\frac{x}{n} \sum_{\substack{a+b=\ell, a, b \geq 0}} \frac{(-1)^{b} \log ^{a}(x / n)}{a ! 2^{b}}-\frac{(-1)^{\ell}}{2^{\ell}} \frac{n}{x}\right) .
\end{aligned}
$$


We specialize to $\ell=0$ from now on:

$$
\begin{aligned}
\beta_{2,0}(x) & =\sum_{n \leq x}(x / n-n / x)=x H_{0}(x)-x+\{x\}+\frac{b_{2}(x)-b_{2}(\{x\})}{2 x} \\
& =x \log x+x\left(\gamma_{0}-\frac{1}{2}\right)-\frac{1}{12 x}-\frac{\{x\}^{2}-\{x\}}{x}+x \varepsilon_{6,0}(x) .
\end{aligned}
$$

This equation implies that $\varepsilon_{6,0}(1)-\frac{1}{12}=\frac{1}{2}-\gamma_{0}$. We thus have

$\sum_{n \leq x} \mu(n)\left(\frac{x \log (x / n)}{n}+\frac{x}{n}\left(\gamma_{0}-\frac{1}{2}\right)-\frac{n}{12 x}-\frac{\{x / n\}^{2}-\{x / n\}}{x / n}+\frac{x}{n} \varepsilon_{6,0}(x / n)\right)=x-x^{-1}$.

Recall [2, (8)]:

$$
\sum_{n \leq x} \mu(n)\left(\frac{x}{n}-1-\frac{\{x / n\}^{2}-\{x / n\}}{x / n}\right)=2-2 x^{-1} \quad(x \geq 1)
$$

and get

$$
\sum_{n \leq x} \mu(n)\left(\frac{x \log (x / n)}{n}+\frac{x}{n}\left(\gamma_{0}-\frac{3}{2}\right)+1-\frac{n}{12 x}+\frac{x}{n} \varepsilon_{6,0}(x / n)\right)=x-2+x^{-1} .
$$

Let us continue the main proof. We get

Lemma 3.2. When $x \geq 1$, we have

$$
\check{m}(x)-1=\frac{6-8 \gamma_{0}}{3 x}-\frac{6-4 \gamma_{0}}{x^{2}}+\frac{6-4 \gamma_{0}}{3 x^{4}}-\frac{1}{x} \int_{1}^{x} M(x / t) h^{\prime}(t) d t
$$

where $h^{\prime}(t)=\left(\frac{3}{2}-\gamma_{0}\right) \varepsilon_{1}^{\prime}(t)+g^{\prime}(t)$ is continuous and differentiable except at integers where it has left and right derivatives. It satisfies

$$
0 \leq t^{2} h^{\prime}(t) \leq \frac{7}{4}-\gamma_{0} .
$$

Theorem 1.4 is a straightforward consequence of this lemma. Let us specify that $h^{\prime}$ is typically not continuous at integer points.

Proof. Indeed, we have already reached

$$
\check{m}(x)-1=\left(\frac{3}{2}-\gamma_{0}\right) m(x)-\left(\frac{3}{2}-\gamma_{0}\right) \frac{M(x)}{x}-\frac{2}{x}+\frac{1}{x^{2}}-\frac{1}{x} \int_{1}^{x} M(x / t) g^{\prime}(t) d t
$$

where the continuous and piecewise differentiable function $g$ is defined by

$$
g(x)=\frac{-1}{12 x}+x \varepsilon_{6,0}(x) \quad\left(g(1)=\frac{1}{2}-\gamma_{0}\right) .
$$

We further recall (2.1):

$$
m(x)=\frac{M(x)}{x}+\frac{4\left(1-x^{-1}\right)^{2}}{x}-\frac{4\left(1-x^{-1}\right)^{3}}{3 x}+\frac{1}{x} \int_{1}^{x} M(x / t) \varepsilon_{1}^{\prime}(t) d t .
$$

This leads to

$$
\begin{aligned}
x(\check{m}(x)-1)=2-\frac{8}{3} \gamma_{0}-\frac{2\left(3-2 \gamma_{0}\right)}{x}+ & \frac{2-\frac{4}{3} \gamma_{0}}{x^{3}} \\
& -\int_{1}^{x} M(x / t)\left(\left(\frac{3}{2}-\gamma_{0}\right) \varepsilon_{1}^{\prime}(t)+g^{\prime}(t)\right) d t .
\end{aligned}
$$

With the notation

$$
h^{\prime}(t)=\left(\frac{3}{2}-\gamma_{0}\right) \varepsilon_{1}^{\prime}(t)+g^{\prime}(t)
$$


we find that (with $u=\{x\}$ )

$$
\begin{aligned}
t^{2} h^{\prime}(t)= & -u^{2}+u-\frac{1}{2}+\left(\frac{3}{2}-\gamma_{0}\right)(2 u-1)^{2} \\
& +t^{2} \int_{t}^{\infty} \frac{B_{2}(v) d t}{v^{3}}+\left(3-2 \gamma_{0}\right) \frac{(2 u-1)\left(u-u^{2}\right)}{t}+\left(\frac{3}{2}-\gamma_{0}\right) \frac{\left(u-u^{2}\right)^{2}}{t^{2}} .
\end{aligned}
$$

The polynomial in $u$ of the first line is positive between 0 and 1 , while the second part is $\ll 1 / x$. Some straightforward numerical work yields $h^{\prime}(t)>0$. On the other hand we recalled after (2.3) that $0 \leq \varepsilon^{\prime}(t) \leq 1 / t^{2}$. Concerning $g^{\prime}(t)$, we first notice that $\left|B_{2}(v)\right| \leq 1 / 6$, so that

$$
\left|t^{2} g^{\prime}(t)\right| \leq\left|\{t\}^{2}-\{t\}+\frac{1}{12}\right|+\frac{1}{6 \times 2} \leq 1 / 4
$$

The lemma readily follows.

\section{Proof of Theorem 1.5] and of Corollary 1.6}

Lemma 4.1. For any positive integer $k$ we have

$$
\int_{k}^{k+1} B_{4}(t) d t / t^{4} \leq 0
$$

Proof. We have, by using the shortcut $u=\{t\}$,

$$
\begin{aligned}
B_{4}(t) & =u^{4}-2 u^{3}+u^{2}-\frac{1}{30} \\
& =t^{4}-(4 k+2) t^{3}+\left(6 k^{2}+6 k+1\right) t^{2}-(4 k+2)\left(k^{2}+k\right) t+\left(k^{2}+k\right)^{2}-\frac{1}{30} .
\end{aligned}
$$

As a consequence and on denoting by $I_{k}$ the integral to be computed, we find that

$$
\begin{aligned}
I_{k}=1 & -2(2 k+1) \log \frac{k+1}{k}+\frac{6 k^{2}+6 k+1}{k(k+1)}-\frac{(2 k+1)^{2}}{k(k+1)} \\
& +\frac{\left(k^{4}+2 k^{3}+k^{2}-\frac{1}{30}\right)\left(3 k^{2}+3 k+1\right)}{3 k^{3}(k+1)^{3}} \\
= & -\frac{1}{63 k^{6}}-\frac{19}{42 k^{7}}+\frac{49}{90 k^{8}}+\mathcal{O}\left(1 / k^{9}\right) .
\end{aligned}
$$

We readily convert this asymptotic expression into an explicit inequality. It finally remains to check the inequality for the first few $k$ 's, and this is readily done.

Lemma 3.2 puts us in the same position as when majorising $m(x)$ in Section 3 , We thus proceed in a similar way. We first notice that, when $n$ is a positive integer,

$$
\begin{aligned}
\int_{n}^{n+1}|M(x / t)| h^{\prime}(t) d t & =\max _{n \leq t \leq n+1}|M(x / t)|(h(n+1)-h(n)) \\
= & \max _{n \leq t \leq n+1}|M(x / t)|\left(\frac{3-2 \gamma_{0}}{6 n}-\frac{3-2 \gamma_{0}}{6(n+1)}+g(n+1)-g(n)\right)
\end{aligned}
$$

and the coefficient of $\max _{n \leq t \leq n+1}|M(x / t)|$ also reads

$$
\begin{aligned}
& \frac{3-\frac{1}{2}-2 \gamma_{0}}{6 n}-\frac{3-\frac{1}{2}-2 \gamma_{0}}{6(n+1)}-n \int_{n}^{\infty} B_{2}(t) \frac{d t}{t^{3}}+(n+1) \int_{n+1}^{\infty} B_{2}(t) \frac{d t}{t^{3}} \\
& \quad=\frac{3-\frac{1}{2}-2 \gamma_{0}}{6 n(n+1)}-n \log \frac{n+1}{n}+\frac{2 n+1}{2(n+1)}-\frac{2 n+1}{12 n(n+1)^{2}}+\int_{n+1}^{\infty} B_{2}(t) \frac{d t}{t^{3}}
\end{aligned}
$$


We now notice that

$$
\int_{n+1}^{\infty} B_{2}(t) \frac{d t}{t^{3}}=\int_{n+1}^{\infty} B_{3}(t) \frac{d t}{t^{3}}=\frac{1}{30(n+1)^{3}}+\int_{n+1}^{\infty} B_{4}(t) \frac{d t}{t^{4}} \leq \frac{1}{30(n+1)^{3}}
$$

by Lemma 4.1. We finally show that, when $n \geq 60$,

$$
\begin{aligned}
& \frac{\frac{5}{2}-2 \gamma_{0}}{6 n(n+1)}-n \log \frac{n+1}{n}+\frac{2 n+1}{2(n+1)}-\frac{2 n+1}{12 n(n+1)^{2}} \\
& \quad+\frac{1}{30(n+1)^{3}} \leq 0.321 \int_{n}^{n+1} \frac{d t}{t^{2}} .
\end{aligned}
$$

(The constant is decreasing in the lower bound for $n$, provided this lower bound be $\geq 3$, the optimal constant being $\left.\left(5-2 \gamma_{0}\right) / 12=0.32046 \cdots\right)$.

Lemma 4.2. The function to which $x \geq D_{0}=464402$ associates,

$$
0.321 \cdot \log x \int_{x D_{0} /\left(x+D_{0}\right)}^{x / 2} \frac{0.0146 \log u-0.1098}{(\log u)^{2}} \frac{u d u}{(x-u)^{2}}+\frac{18192350 \log x}{x^{2}},
$$

is decreasing between $D_{0}$ and $D_{1}=1.60510 \cdots 10^{6}$ and increasing afterwards to $0.321 \cdot 0.0146(1-\log 2)=0.001438 \cdots$. As a conclusion, it is $\leq 0.00144$ when $x \geq D_{0}$.

Proof. We simply plot the function via

$$
\begin{aligned}
&\{f(x)=\operatorname{intnum}(u=x * D 0 /(x+D 0), x / 2, \\
&\left.(0.0146 * \log (u)-0.1098) * u /\left((\log (u))^{\wedge} 2 *(x-u)^{-} 2\right)\right) \\
&\left.* \log (x) * 0.321+18192350 * \log (x) / x^{\wedge} 2\right\}
\end{aligned}
$$

default (realprecision, 10);

$\operatorname{plot}\left(t=10^{\wedge} 6,10^{\wedge} 8, f(t)\right)$;

Thus, when $x \geq D_{0}$ and recalling Lemma 2.3 we have:

$$
\begin{aligned}
\int_{1}^{x} \mid & M(x / t)\left|h^{\prime}(t) d t \leq \int_{1}^{x / D_{0}}\right| M(x / t) \mid h^{\prime}(t) d t+\left(\frac{7}{4}-\gamma_{0}\right) \frac{15512101}{x} \\
\leq & 0.321 \cdot x \int_{1}^{x / D_{0}} \frac{0.0146 \log (x /(t+1))-0.1098}{(\log (x /(t+1)))^{2}} \frac{d t}{t^{2}(t+1)}+\frac{18192350}{x} \\
& \leq 0.321 \cdot x \int_{x D_{0} /\left(x+D_{0}\right)}^{x / 2} \frac{0.0146 \log u-0.1098}{(\log u)^{2}} \frac{u d u}{(x-u)^{2}}+\frac{18192350}{x} \\
& \leq 0.00144 x / \log x
\end{aligned}
$$

by Lemma 4.2. We then use Lemma 3.2 (notice that $\frac{1}{2}-\gamma_{0}<0$ ). This yields

$$
\begin{aligned}
|\check{m}(x)-1| \leq\left(\gamma_{0}-\frac{1}{2}\right) \frac{0.0146 \log x-0.1098}{(\log x)^{2}}+\frac{2}{x}+\frac{0.00144}{\log x} & \\
\leq & \leq \frac{0.00257 \log x-0.0077}{(\log x)^{2}}
\end{aligned}
$$

when $x \geq D_{0}$. We then appeal to Lemma 10.2 to extend the range to $x \geq 13950$ and checked numerically its extension to the range $3846 \leq x \leq 10^{6}$. Theorem 1.5 
follows readily. The GP-script is called AsymptoticBoundsFor_checkm.gp and the main function is getboundsbisaux.

The proof of Corollary 1.6 is immediate.

\section{An IDENTITY FOR $\check{m}(x)$ AND PROOF OF Theorem 1.7}

We start with (3.4) with the choice $\ell=1$ and using (3.1) we have:

$$
\begin{aligned}
\beta_{2,1}(x)= & \sum_{n \leq x}\left(\frac{x}{n}\left(\log (x / n)-\frac{1}{2}\right)+\frac{n}{2 x}\right) \\
= & x \log (x / e) H_{0}(x)+\frac{x}{2} H_{0}(x)-x H_{1}(x)+\frac{x}{4}-\frac{B_{1}(x)}{2}+\frac{B_{2}(x)}{4 x}-\frac{1}{24 x} \\
= & \frac{x \log ^{2} x}{2}+x\left(\gamma_{0}-\frac{1}{2}\right) \log x+x\left(-\frac{1}{2} \gamma_{0}-\gamma_{1}+\frac{1}{4}\right) \\
& +\frac{B_{2}(x)}{4 x}-\frac{1}{24 x}+x\left(\log x-\frac{1}{2}\right) \varepsilon_{6,0}(x)-x \varepsilon_{6,1}(x) .
\end{aligned}
$$

We note here, that by construction, (cf. (3.3)), the error term above is continuous all over, and continuously differentiable except maybe at the integer point, where it has left and right derivatives. Furthermore, $-\frac{1}{2} \varepsilon_{6,0}(1)-\varepsilon_{6,1}(1)-\frac{1}{2} \gamma_{0}-\gamma_{1}+\frac{1}{4}=0$. The above equation implies that

$$
\begin{aligned}
\sum_{n \leq x} \mu(n) & \left(\frac{x \log ^{2}(x / n)}{2 n}+\frac{x}{n}\left(\gamma_{0}-\frac{1}{2}\right) \log \frac{x}{n}-\frac{x}{n}\left(\frac{1}{2} \gamma_{0}+\gamma_{1}-\frac{1}{4}\right)\right. \\
+\frac{\{x / n\}^{2}-\{x / n\}}{4 x / n}+\frac{x\left(\log (x / n)-\frac{1}{2}\right)}{n} \varepsilon_{6,0}(x / n)- & \left.\frac{x}{n} \varepsilon_{6,1}(x / n)\right) \\
& =x \log x-\frac{x}{2}+\frac{1}{2 x} .
\end{aligned}
$$

We again use (3.5) in the form

$$
\sum_{n \leq x} \mu(n) \frac{\{x / n\}^{2}-\{x / n\}}{x / n}=-2+2 x^{-1} \sum_{n \leq x} \mu(n)\left(\frac{x}{n}-1\right)
$$

to get

$$
\begin{aligned}
\sum_{n \leq x} \mu(n)\left(\frac{x \log ^{2}(x / n)}{2 n}+\frac{x}{n}\left(\gamma_{0}-\frac{1}{2}\right) \log \frac{x}{n}-\frac{x}{n}\left(\frac{1}{2} \gamma_{0}+\gamma_{1}+\frac{1}{4}\right)\right. & \\
\left.+\frac{1}{2}+\frac{x\left(\log (x / n)-\frac{1}{2}\right)}{n} \varepsilon_{6,0}(x / n)-\frac{x}{n} \varepsilon_{6,1}(x / n)\right) & =x \log x-\frac{x}{2}+\frac{1}{x}-\frac{1}{2} .
\end{aligned}
$$

Here is our main lemma:

Lemma 5.1. When $x \geq 1$, we have

$$
\check{\check{m}}(x)-2 \log x+2 \gamma_{0}=2\left(\gamma_{0}-\frac{1}{2}\right)^{2} \frac{M(x)}{x}+\frac{1}{x} \int_{1}^{x} M(x / t) k_{2}^{\prime}(t) d t+\frac{K_{2}(1 / x)}{x}
$$

where

$$
K_{2}(v)=1+4 \gamma_{0}+\left(3-2 \gamma_{0}\right) v-\left(8 \gamma_{0}^{2}-12 \gamma_{0}+2 \gamma_{1}+2\right) \frac{(1-v)(2+v)}{3}
$$


satisfies $4.5 \leq K_{2}(v) \leq 4+2 \gamma_{0}$ when $0 \leq v \leq 1$ and

$$
k_{2}^{\prime}(t)=\left(2 \gamma_{0}^{2}-3 \gamma_{0}+2 \gamma_{1}+\frac{1}{2}\right) \varepsilon_{1}(t)+2\left(\gamma_{0}-\log t\right) t \varepsilon_{6,0}(t)+2 t \varepsilon_{6,1}(t)-\frac{2 \gamma_{0}-1}{12 t}
$$

is continuous and differentiable except at integers where it has left and right derivatives. It satisfies

$$
t^{2}\left|k_{2}^{\prime}(t)\right| \leq 1.46
$$

Theorem 1.7 is a straightforward consequence of this lemma. Let us specify that $h^{\prime}$ is typically not continuous at integer points.

Proof. Indeed, we have already reached

$$
\begin{aligned}
\frac{1}{2} \check{\check{m}}(x)+\left(\gamma_{0}-\frac{1}{2}\right)(\check{m}(x)-1)- & \left(\frac{1}{2} \gamma_{0}+\gamma_{1}+\frac{1}{4}\right)\left(m(x)-M(x) x^{-1}\right) \\
+ & \frac{1}{x} \int_{1}^{x} M(x / t) q^{\prime}(t) d t=\log x-\gamma_{0}+\frac{1}{x^{2}}-\frac{1}{2 x}
\end{aligned}
$$

with the notation

$$
q(t)=t\left(\log t-\frac{1}{2}\right) \varepsilon_{6,0}(t)-t \varepsilon_{6,1}(t), \quad\left(q(1)=-\frac{1}{2} \gamma_{0}+\gamma_{1}\right) .
$$

During the proof of Lemma (3.2), we have noticed that

$$
\check{m}(x)-1=\left(\frac{3}{2}-\gamma_{0}\right) m(x)-\frac{M(x)}{x}-\frac{2}{x}+\frac{1}{x^{2}}-\frac{1}{x} \int_{1}^{x} M(x / t) g^{\prime}(t) d t
$$

where the function $g$ is defined by

$$
g(t)=\frac{-1}{12 t}+t \varepsilon_{6,0}(t)
$$

Combining both identities leads to

$$
\begin{aligned}
& \frac{1}{2} \check{m}(x)+\left(-\gamma_{0}^{2}+\frac{3}{2} \gamma_{0}-\gamma_{1}-\frac{1}{4}\right)\left(m(x)-M(x) x^{-1}\right)-\left(\gamma_{0}-\frac{1}{2}\right)^{2} \frac{M(x)}{x} \\
& +\frac{1}{x} \int_{1}^{x} M(x / t)\left(q^{\prime}(t)-\left(\gamma-\frac{1}{2}\right) g^{\prime}(t)\right) d t=\log x-\gamma_{0}+\frac{1+4 \gamma_{0}}{2 x}+\frac{3-2 \gamma_{0}}{2 x^{2}} .
\end{aligned}
$$

We further recall (2.1):

$$
m(x)-\frac{M(x)}{x}=\frac{4\left(1-x^{-1}\right)^{2}}{x}-\frac{4\left(1-x^{-1}\right)^{3}}{3 x}+\frac{1}{x} \int_{1}^{x} M(x / t) \varepsilon_{1}^{\prime}(t) d t
$$

and thus

$$
\check{\check{m}}(x)-2 \log x+2 \gamma_{0}=2\left(\gamma_{0}-\frac{1}{2}\right)^{2} \frac{M(x)}{x}+\frac{1}{x} \int_{1}^{x} M(x / t) k_{2}^{\prime}(t) d t+\frac{K_{2}(1 / x)}{x}
$$

where

$$
k_{2}^{\prime}(t)=-2\left(-\gamma_{0}^{2}+\frac{3}{2} \gamma_{0}-\gamma_{1}-\frac{1}{4}\right) \varepsilon_{1}^{\prime}(t)-2 q^{\prime}(t)+\left(2 \gamma_{0}-1\right) g^{\prime}(t)
$$

and $K_{2}$ is defined in (5.1). We have

$$
k_{2}(t)=\left(2 \gamma_{0}^{2}-3 \gamma_{0}+2 \gamma_{1}+\frac{1}{2}\right) \varepsilon_{1}(t)+2\left(\gamma_{0}-\log t\right) t \varepsilon_{6,0}(t)+2 t \varepsilon_{6,1}(t)-\frac{2 \gamma_{0}-1}{12 t} .
$$

Let us recall the definitions of $\varepsilon_{6,0}$ and $\varepsilon_{6,1}$ :

$$
\varepsilon_{6,0}(x)=\int_{x}^{\infty} B_{2}(t) \frac{d t}{t^{3}}, \quad \varepsilon_{6,1}(x)=\frac{1}{2} \int_{x}^{\infty} B_{2}(t) \frac{(2 \log t+1) d t}{t^{3}} .
$$


We deduce from that (when $x$ is not an integer):

$$
\varepsilon_{6,0}^{\prime}(x)=-\frac{B_{2}(x)}{x^{3}}, \quad \varepsilon_{6,1}^{\prime}(x)=-\frac{B_{2}(x)(2 \log x+1)}{2 x^{3}} .
$$

This leads to the following expression for $k_{2}^{\prime}(t)$ :

$$
\begin{aligned}
t^{2} k_{2}^{\prime}(t)=\left(2 \gamma_{0}^{2}-\right. & \left.3 \gamma_{0}+2 \gamma_{1}+\frac{1}{2}\right) t^{2} \varepsilon_{1}^{\prime}(t)-2\left(2 \gamma_{0}+1\right) B_{2}(t)+\frac{2 \gamma_{0}-1}{12} \\
& +2\left(\gamma_{0}-\log t\right) t^{2} \int_{t}^{\infty} B_{2}(u) \frac{d u}{u^{3}}+t^{2} \int_{t}^{\infty} B_{2}(u) \frac{(2 \log u+1) d u}{u^{3}} .
\end{aligned}
$$

An integration by parts using $B_{3}^{\prime}(t)=3 B_{2}(t)$ (where $b_{3}(x)=x^{3}-\frac{3}{2} x^{2}+\frac{1}{2} x$ and $\left.B_{3}(t)=b_{3}(\{t\})\right)$ gives us

$$
\begin{aligned}
t^{2} k_{2}^{\prime}(t) & =\left(2 \gamma_{0}^{2}-3 \gamma_{0}+2 \gamma_{1}+\frac{1}{2}\right) t^{2} \varepsilon_{1}^{\prime}(t)-2\left(2 \gamma_{0}+1\right) B_{2}(t)+\frac{2 \gamma_{0}-1}{12} \\
- & \left(1+2 \gamma_{0}\right) \frac{B_{3}(t)}{3 t}+\frac{6 \gamma_{0}+1}{3} t^{2} \int_{t}^{\infty} B_{3}(u) \frac{d u}{u^{4}}+2 t^{2} \int_{t}^{\infty} B_{3}(u) \frac{\log (u / t)}{u^{4}} d u .
\end{aligned}
$$

We find that the part without the last two integrals equals (on using $u=\{t\}$ )

$$
\begin{aligned}
\left(2 \gamma_{0}^{2}-3 \gamma_{0}+2 \gamma_{1}+\frac{1}{2}\right) \frac{\left((2 u-1) t+u-u^{2}\right)^{2}}{t^{2}} & -\left(4 \gamma_{0}+2\right)\left(u^{2}-u+\frac{1}{6}\right) \\
+ & \frac{2 \gamma_{0}-1}{12}-\left(1+2 \gamma_{0}\right) \frac{2 u^{3}-3 u^{2}+u}{6 t}
\end{aligned}
$$

with $\gamma_{1}=-0.07281584548 \cdots$. Some numerical analysis tells us that this function is increasing then decreasing between two consecutive integers. It takes its minimal value there and this value is:

$2 \gamma_{0}^{2}-3 \gamma_{0}+2 \gamma_{1}+\frac{1}{2}-\frac{2 \gamma_{0}+1}{3}+\frac{2 \gamma_{0}-1}{12}=2 \gamma_{0}^{2}-\frac{7}{2} \gamma_{0}+2 \gamma_{1}+\frac{1}{12}=-1.41687 \cdots$.

The local maxima are all not more than 0.38 . The function $B_{3}$ satisfies $B_{3}(1-t)=$ $-B_{3}(t)$. It attains its maximum at $(3-\sqrt{3}) / 6$ and minimum at $(3+\sqrt{3}) / 6$. The value of this maximum is $\sqrt{3} / 36=0.04811 \cdots$. As a consequence

$$
\begin{aligned}
\frac{6 \gamma_{0}+1}{3} t^{2} \int_{t}^{\infty}\left|B_{3}(u)\right| \frac{d u}{u^{4}} & +2 t^{2} \int_{t}^{\infty}\left|B_{3}(u)\right| \frac{\log (u / t)}{u^{4}} d u \\
& \leq \frac{6 \gamma_{0}+1}{3} \frac{\sqrt{3}}{36} \frac{1}{3 t}+\frac{\sqrt{3}}{36} \frac{2}{t} \int_{1}^{\infty} \frac{\log v}{v^{4}} d v=\left(2 \gamma_{0}+1\right) \frac{\sqrt{3}}{108 t} .
\end{aligned}
$$

The part concerning $k_{2}^{\prime}$ follows readily. It is easy to study $K_{2}(u)$ and show the bounds claimed. The maximum is attained at $u=1$.

\section{Proof of Theorem 1.8}

By using Lemma 5.1 together with Lemma 2.1. we find that with $D_{0}=1078853$,

$$
\begin{aligned}
\left|\check{m}(x)-2 \log x+2 \gamma_{0}\right| \leq & 2\left(\gamma_{0}-\frac{1}{2}\right)^{2} \frac{|M(x)|}{x}+\frac{1.46}{x^{2}} \int_{1}^{x}|M(t)| d t+\frac{5.2}{x} \\
\leq & 2\left(\gamma_{0}-\frac{1}{2}\right)^{2} \frac{0.0130 \log x-0.118}{(\log x)^{2}}+\frac{1.46}{x^{2}} \int_{1}^{D_{0}}|M(t)| d t \\
& +\frac{1.46}{x^{2}} \int_{D_{0}}^{x} \frac{0.0130 \log t-0.118}{(\log t)^{2}} t d t+\frac{5.2}{x} .
\end{aligned}
$$


We have

$$
\begin{gathered}
\int_{D_{0}}^{x} \frac{0.0130 \log t-0.118}{(\log t)^{2}} t d t=\frac{0.0130 x^{2}}{2 \log x}-\frac{0.0130 D_{0}^{2}}{2 \log D_{0}}-\int_{D_{0}}^{x} \frac{0.118-\frac{0.0130}{2}}{(\log t)^{2}} t d t \\
=\frac{0.0130 x^{2}}{2 \log x}-\frac{0.0130 D_{0}^{2}}{2 \log D_{0}}-\frac{0.1115 x^{2}}{2(\log x)^{2}}-\frac{0.1115 D_{0}^{2}}{2\left(\log D_{0}\right)^{2}}-\int_{D_{0}}^{x} \frac{0.1115 t}{(\log t)^{3}} d t \\
\leq \frac{0.0130 x^{2}}{2 \log x}-\frac{0.0130 D_{0}^{2}}{2 \log D_{0}}-\frac{0.1115 x^{2}}{2(\log x)^{2}}-\frac{0.1115 D_{0}^{2}}{2\left(\log D_{0}\right)^{2}}
\end{gathered}
$$

Moreover, GP/PARI tells us (see script CompIntM.gp of Lemma 2.2) that

$$
\int_{1}^{1100000}|M(t)| d t=118577036
$$

and

$$
118577036-\frac{0.0130 D_{0}^{2}}{2 \log D_{0}}-\frac{0.1115 D_{0}^{2}}{2\left(\log D_{0}\right)^{2}} \leq-10^{9} .
$$

We have thus reached:

$$
\begin{aligned}
\left|\check{\check{m}}(x)-2 \log x+2 \gamma_{0}\right| \leq & 2\left(\gamma_{0}-\frac{1}{2}\right)^{2} \frac{0.0130 \log x-0.118}{(\log x)^{2}} \\
& +1.46 \frac{0.0130 \log x-0.1115}{2(\log x)^{2}}+\frac{5.2}{x} \\
\leq & \frac{0.00965 \log x-0.0818}{(\log x)^{2}} .
\end{aligned}
$$

We check numerically this bound with Gp/Pari for $10 \leq x \leq 10^{7}$. The script used is AsymptoticBoundsFor_checkcheckm.gp, function getboundsteraux.

\section{An INTERMEDiATE FUNCTION FOR $m$}

In this part, we produce upper bounds for

$$
m^{*}(y)=\sum_{w \leq \sqrt{y+1}} \max _{y / w^{2} \leq z<(y+1) / w^{2}}|m(z)| / w^{2} .
$$

We have taken the maximum so that we may restrict our attention to integer values of $y$. Notice that the interval $\left[y / w^{2},(y+1) / w^{2}\right)$ contains at most one integer.

Lemma 7.1. For integer $x$, we have

$$
m^{*}(x) \sqrt{x} / \sqrt{1+\log x} \leq \begin{cases}0.5 & \text { when } x \in[171454,33000000] \\ 0.557 & \text { when } x \in[100,33000000] \\ 0.578 & \text { when } x \in[1,33000000]\end{cases}
$$

We precomputed the values of $\mu(n)$ for $n$ up to $3.3 \cdot 10^{7}$, then the values of $m(x)$ for $x$ up to $3.3 \cdot 10^{7}$. The script is called AsymptoticBoundsFor_mstar.gp, its main function being getboundsmstar and the total run time has been about 25 hours on the material specified in the introduction.

We compared $\left|m^{*}(x)\right|$ with $\sqrt{1+\log x} / \sqrt{x}$ as this seems coherent with the numerical outputs: the successive maxima seemed to be oscillating in the neighbourhood $0.5 \sqrt{1+\log x} / \sqrt{x}$ and slightly dipping when $x$ grows. The numerical data is, however, way too thin to allow any conjecture. It seems clear that the work [17. 
can be adapted to $m(x)$, but no such straightforward heuristic can be deduced for $m^{*}(x)$.

Here is a generalization of [19, Lemma 2.2]:

Lemma 7.2. Let $A>$ e be a given parameter. The function

$$
T(y): y \mapsto \frac{\log y}{y} \int_{A}^{y} \frac{d v}{\log v}
$$

is first increasing and then decreasing. It reaches its maximum at $y_{0}(A)$ where $y_{0}(A)$ is the unique solution of $y=(\log y-1) \int_{A}^{y} d v / \log v$. Moreover, we have $T\left(y_{0}(A)\right)=\left(\log y_{0}(A)\right) /\left(\log y_{0}(A)-1\right)$.

Proof. We compute its derivative and get

$$
\frac{y^{2}}{\log y-1} T^{\prime}(y)=\frac{y}{\log y-1}-\int_{A}^{y} \frac{d v}{\log v}=h(y),
$$

say. We check that $h^{\prime}(y)=-1 /\left[\log y(\log y-1)^{2}\right]$. We have $h(A)>0$ and $h$ tends to be negative in the vicinity of infinity. Indeed, after several integration by parts, we reach

$$
\begin{aligned}
h(y)=\frac{y}{\log y-1}-\frac{y}{\log y}-\frac{y}{\log ^{2} y}- & \frac{2 y}{\log ^{3} y} \\
& +\frac{A}{\log A}+\frac{A}{\log ^{2} A}+\frac{2 A}{\log ^{3} A}-6 \int_{A}^{y} \frac{d v}{\log ^{4} v} .
\end{aligned}
$$

Lemma 7.3. With $X_{0}=1426$, the function

$$
T(y): y \mapsto \frac{\log y}{y} \int_{\sqrt{X_{0}}}^{y} \frac{d v}{\log v}
$$

is increasing and then decreasing, reaching a maximum around 144.803 with value $1.251548+\mathcal{O}^{*}\left(10^{-6}\right)$. Moreover, $T\left(\sqrt{3.3 \cdot 10^{7}}\right) \leq 1.139$.

Lemma 7.4. For $x \geq 11808$, we have $\left|m^{*}(x)\right| \leq 0.132 / \log x$.

For $x \geq 687$, we have $\left|m^{*}(x)\right| \leq 0.320 / \log x$.

For $x>1$, we have $\left|m^{*}(x)\right| \leq 0.779 / \log x$.

Proof. The initial step is provided by Theorem 1.2 .

$$
|m(x)| \leq 1 /(20 \log x) \quad\left(x \geq X_{0}=1426\right) .
$$

Thus, for $x / U^{2}=X_{0}$,

$$
\left|m^{*}(x)\right| \leq \frac{1}{20} \sum_{u \leq U} \frac{1}{u^{2} \log \left(x / u^{2}\right)}+\frac{\max _{U<u \leq U+1}\left|m\left(x / u^{2}\right)\right|}{U^{2}}+\int_{U}^{\infty} \frac{\left|m\left(x / u^{2}\right)\right| d u}{u^{2}} .
$$


We appeal to Corollary 1.3 and get that the sum of the last terms is bounded above by

$$
\begin{aligned}
\frac{1}{100 U^{2}} & +\int_{U}^{\sqrt{x / 694}} \frac{d u}{100 u^{2}}+\int_{\sqrt{x / 694}}^{\sqrt{x / 41}} \frac{d u}{20 u^{2}}+\int_{\sqrt{x / 41}}^{\infty} \frac{d u}{u^{2}} \\
& \leq \frac{1}{U}\left(\frac{1}{100 U}+\frac{1}{100}+\frac{U}{20 \sqrt{x / 694}}+\frac{U}{\sqrt{x / 41}}\right) \\
& \leq \frac{1}{U}\left(\frac{1}{100 \sqrt{x / X_{0}}}+\frac{1}{100}+\frac{1}{20 \sqrt{X_{0} / 694}}+\frac{1}{\sqrt{X_{0} / 41}}\right) \leq 0.215 / U .
\end{aligned}
$$

We continue by using a comparison with an integral

$$
\begin{aligned}
\left|m^{*}(x)\right| & \leq \frac{1}{20} \sum_{u \leq U} \frac{1}{u^{2} \log \left(x / u^{2}\right)}+\frac{0.215}{U} \\
& \leq \frac{1}{20 \log x}+\frac{1}{20 \sqrt{x}} \int_{\sqrt{x / U^{2}}}^{\sqrt{x}} \frac{d v}{2 \log v}+\frac{0.205}{U} \\
& \leq \frac{1}{20 \log x}+\frac{1}{20 \sqrt{x}} \int_{\sqrt{X_{0}}}^{\sqrt{x}} \frac{d v}{2 \log v}+\frac{0.215}{\sqrt{x / X_{0}}} .
\end{aligned}
$$

We employ Lemma 7.3 at this level. Hence, when $x \geq 3.3 \cdot 10^{7}$,

$$
\left|m^{*}(x)\right| \leq \frac{1}{20 \log x}+\frac{1.139}{20 \log x}+\frac{0.215}{\sqrt{x / X_{0}}} \leq \frac{0.132}{\log x} .
$$

We extend it to $x \geq 21500$ by using Lemma 7.1. We reduce this bound by direct verification by adapting the script AsymptoticBoundsFor_mstar.gp, function getboundsmstar.

\section{An intermediate FUnCTION FOR $m$, Bis REPEtita}

The previous section was dedicated to getting bounds of the shape $1 / \log x$ and we aim here at bounds of the shape $1 /(1+\log x)$. There are no difficulties, but the computations need to be written down.

Here is another version of Lemma 8.1 .

Lemma 8.1. Let $A>$ e be a given parameter. The function

$$
T^{*}(y): y \mapsto \frac{1+\log y}{y} \int_{A}^{y} \frac{d v}{\log v}
$$

is first increasing and then decreasing. It has a maximum at $y^{*}(A)$ where $y^{*}(A)$ is the solution of $(1+\log y) y=(\log y)^{2} \int_{A}^{y} d v / \log v$ and we have $T^{*}\left(y^{*}(A)\right)=$ $\left(1+\log y^{*}(A)\right)^{2} /\left(\log y^{*}(A)\right)^{2}$.

Proof. We compute its derivative and get

$$
\frac{y^{2}}{\log y} T^{* \prime}(y)=\frac{y(1+\log y)}{(\log y)^{2}}-\int_{A}^{y} \frac{d v}{\log v}=h^{*}(y),
$$

say. We check that $h^{* \prime}(y)=-2 /(\log y)^{3}$. We have $h^{*}(A)>0$ and $h^{*}$ tends to be negative in the vicinity of infinity. Indeed, after several integrations by parts, we reach

$$
h^{*}(y)=\frac{A}{\log A}+\frac{A}{\log ^{2} A}-2 \int_{A}^{y} \frac{d v}{\log ^{3} v} .
$$


Lemma 8.2. With $X_{0}=1426$, the function

$$
T^{*}(y): y \mapsto \frac{1+\log y}{y} \int_{\sqrt{X_{0}}}^{y} \frac{d v}{\log v}
$$

is increasing and then decreasing, reaching a maximum around 1613.183 with value $1.289114+\mathcal{O}^{*}\left(10^{-6}\right)$. Moreover, $T^{*}\left(\sqrt{3.3 \cdot 10^{7}}\right) \leq 1.28$.

Lemma 8.3. For $x \geq 11811$, we have $\left|m^{*}(x)\right| \leq 0.143 /(1+\log x)$.

For $x \geq 882$, we have $\left|m^{*}(x)\right| \leq 0.320 /(1+\log x)$.

For $x \geq 296$, we have $\left|m^{*}(x)\right| \leq 0.5 /(1+\log x)$.

For $x>1$, we have $\left|m^{*}(x)\right| \leq 1.694 /(1+\log x)$.

Proof. We follow the proof of Lemma 7.4 until (7.3) which we modify into

$$
\left|m^{*}(x)\right| \leq \frac{1}{20 \log x}+\frac{1.28}{25(1+\log x)}+\frac{0.215}{\sqrt{x / X_{0}}} \leq \frac{0.143}{1+\log x} .
$$

We extend it to $x \geq 22100$ by using Lemma 7.1. We reduce this bound by direct verification by again adapting the script AsymptoticBoundsFor_mstar.gp, function getboundsmstar.

\section{Proof of Theorems 1.12 and 1.13}

We proceed to prove the estimate concerning $m_{q}(x)$. Lemmas 1.9 and 7.4 give us, for a real parameter $U$ such that $x / q \geq X_{1}$,

$$
\begin{aligned}
\left|m_{q}(x)\right| & \leq \sum_{d \mid q} \frac{\mu^{2}(d)}{d} m^{*}(x / d) \\
& \leq \frac{q}{\varphi(q) \log (x / q)} \begin{cases}0.132 & \text { when } x / q \geq 11808, \\
0.320 & \text { when } x / q \geq 687, \\
0.779 & \text { when } x / q>1 .\end{cases}
\end{aligned}
$$

Theorem 1.12 follows readily. We proceed in a similar way with Theorem 1.13 but on using Lemma 8.3 .

\section{AN INTERMEDIATE FUNCTION FOR $\check{m}$}

In this part, we produce upper bounds for

$$
\check{m}^{*}(y)=\sum_{w \leq \sqrt{y+1}} \max _{y / w^{2} \leq z<(y+1) / w^{2}}|\check{m}(z)-1| / w^{2} .
$$

We have again taken the maximum so that we may restrict our attention to integer values of $y$. With such a definition, our script will spend most of its time computing logarithms, which are costly. Let us investigate somewhat further what happens. The interval is $\left[y / w^{2},(y+1) / w^{2}\right)$. If there are no integers lying in this interval, then let us set

$$
A=\sum_{n \leq y / w^{2}} \mu(n) / n, \quad B=-1+\sum_{n \leq y / w^{2}} \mu(n)(\log n) / n .
$$

The function $|A \log z-B|$ is maximized at $\log z=\log y-2 \log w$ or at $\log z=$ $\log (y+1)-2 \log w$. If there is an integer, say $m$ lying in this interval, then we have to maximize $|A \log z-B|$ when $z \in\left[y / w^{2}, m\right)$ and a similar expression when 
$z \in\left[n, y /(w+1)^{2}\right)$. In both cases, we can recover the relevant $\log z$ in terms of the logarithms of integers $\leq y$. It is thus enough to precompute these.

Lemma 10.1. For integer $x$, we have

$$
\check{m}^{*}(x) \sqrt{x} \leq \begin{cases}0.440 & \text { when } x \in[64,12000000] \\ 0.504 & \text { when } x \in[16,12000000] \\ 1 & \text { when } x \in[1,12000000]\end{cases}
$$

We precomputed the values of $\mu(n)$ and $\log n$ for $n$ up to $1.2 \cdot 10^{7}$, then the values of $m(x)$ and of $-1+\sum_{n \leq x} \mu(n)(\log n) / n$ for $x$ up to $1.2 \cdot 10^{7}$. The total run time has been about 8 hours. The constant 0.440 comes from the behaviour of $\check{m}^{*}(x)$ for $x \in$ [9 400 000, 9600 000]. The script is called AsymptoticBoundsFor_checkmstar.gp, and its main function is called getboundscheckmstar

We compared $\left|\check{m}^{*}(x)\right|$ to $1 / \sqrt{x}$ as this seems coherent with the numerical outputs; the quantity $\check{m}^{*}(x) \sqrt{x}$ oscillates between 0.425 and 0.439 , this maximum increasing slightly with $x$.

We used a very similar scheme to show that

Lemma 10.2. We have, for real $x$,

$$
|\check{m}(x)-1| \sqrt{x} \leq \begin{cases}0.0192 & \text { when } 340000 \leq x \leq 12000000 \\ 0.0218 & \text { when } 11 \leq x \leq 12000000 \\ 1 & \text { when } 1 \leq x \leq 12000000\end{cases}
$$

Another numerical application of Lemma 7.2 yields:

Lemma 10.3. With $Y_{0}=16$, the function

$$
T(y): y \mapsto \frac{\log y}{y} \int_{\sqrt{Y_{0}}}^{y} \frac{d v}{\log v}
$$

is increasing and then decreasing, reaching a maximum around 139.45 with value $1.253951+\mathcal{O}^{*}\left(10^{-6}\right)$.

Lemma 10.4. We have, for real $x$,

$$
\left|\check{m}^{*}(x)\right| \log x \leq \begin{cases}0.0538 & \text { when } x \geq 3158 \\ 0.200 & \text { when } x \geq 101 \\ 0.250 & \text { when } x>1\end{cases}
$$

Proof. The initial step is provided by Theorem 1.5.

$$
|\check{m}(x)-1| \leq 1 /(389 \log x) \quad\left(x \geq Y_{0}=16\right) .
$$

For smaller $x$, we use $0 \leq \check{m}(x) \leq 1.004$ which implies that $|\check{m}(x)-1| \leq 1$. Thus, for $x / U^{2}=Y_{0}$,

$$
\left|\check{m}^{*}(x)\right| \leq \sum_{u \leq U} \frac{0.0203}{u^{2} \log \left(x / u^{2}\right)}+\frac{\max _{U<u \leq U+1}\left|\check{m}\left(x / u^{2}\right)-1\right|}{U^{2}}+\int_{U}^{\infty} \frac{\left|\check{m}\left(x / u^{2}\right)-1\right| d u}{u^{2}} .
$$


We appeal to Corollary 1.3 and get that the sum of the last terms is bounded above by

$$
\begin{aligned}
\frac{1}{125 U^{2}} & +\int_{U}^{\sqrt{x / 7}} \frac{d u}{125 u^{2}}+\int_{\sqrt{x / 7}}^{\infty} \frac{d u}{u^{2}} \\
& \leq \frac{1}{U}\left(\frac{1}{125 U}+\frac{1}{125}+\frac{U}{\sqrt{x}}\right) \\
& \leq \frac{1}{U}\left(\frac{1}{125 \sqrt{x / Y_{0}}}+\frac{1}{125}+\frac{1}{\sqrt{Y_{0} / 7}}\right) \leq 0.667 / U
\end{aligned}
$$

We continue by using a comparison with an integral

$$
\begin{aligned}
\left|\check{m}^{*}(x)\right| & \leq \sum_{u \leq U} \frac{0.0203}{u^{2} \log \left(x / u^{2}\right)}+\frac{0.667}{U} \\
& \leq \frac{0.0203}{\log x}+\frac{0.0203}{\sqrt{x}} \int_{\sqrt{x / U^{2}}}^{\sqrt{x}} \frac{d v}{2 \log v}+\frac{0.667}{U} . \\
& \leq \frac{0.0203}{\log x}+\frac{0.0203}{\sqrt{x}} \int_{\sqrt{Y_{0}}}^{\sqrt{x}} \frac{d v}{2 \log v}+\frac{0.667}{\sqrt{x / Y_{0}}} .
\end{aligned}
$$

We again employ Lemma 10.3 at this level. Hence, when $x \geq 1.2 \cdot 10^{7}$,

$$
\left|\check{m}^{*}(x)\right| \leq \frac{0.0203}{\log x}+\frac{1.254 \times 0.0203}{\log x}+\frac{0.667}{\sqrt{x / Y_{0}}} \leq \frac{0.0584}{\log x} .
$$

We extend it to $x \geq 3900$ by using Lemma 10.1 and to $x \geq 3158$ by direct checking done by modifying suitably AsymptoticBoundsFor_checkmstar.gp.

\section{An InTERMEdiate FUnCTION FOR $\check{m}$, BIS REPETItA}

The previous section was dedicated to getting bounds of the shape $1 / \log x$ and we aim here at bounds of the shape $1 /(1+\log x)$. There are no difficulties, but the computations need to be written down.

Another numerical application of Lemma 8.1 yields:

Lemma 11.1. With $Y_{0}=16$, the function

$$
T^{*}(y): y \mapsto \frac{1+\log y}{y} \int_{\sqrt{Y_{0}}}^{y} \frac{d v}{\log v}
$$

is increasing and then decreasing, reaching a maximum around 68.49 with value $1.529154+\mathcal{O}^{*}\left(10^{-6}\right)$.

Lemma 11.2. We have, for real $x$,

$$
\left|\check{m}^{*}(x)\right|(1+\log x) \leq \begin{cases}0.0660 & \text { when } x \geq 3150 \\ 0.200 & \text { when } x \geq 171 \\ 1 & \text { when } x>1\end{cases}
$$

Proof. We follow the proof of Lemma 7.4 until (10.3) which we modify into

$$
\left|\check{m}^{*}(x)\right| \leq \frac{0.0203}{\log x}+\frac{1.530 \times 0.0203}{1+\log x}+\frac{0.667}{\sqrt{x / Y_{0}}} \leq \frac{0.0660}{1+\log x} .
$$


We extend it to $x \geq 3800$ by using Lemma 7.1. We reduce this bound by direct verification, again realised by modifying suitably AsymptoticBoundsFor_checkmstar.gp.

\section{Proof of Theorem 1.14 And 1.15}

We proceed to prove the estimate concerning $\check{m}_{q}(x)$. Lemma 1.9 gives us,

$$
\begin{aligned}
\check{m}_{q}(x) & =\sum_{d \mid q} \frac{\mu^{2}(d)}{d} \sum_{\substack{w \leq \sqrt{x / d}, w \mid q^{\infty}}} \check{m}\left(x /\left(d w^{2}\right)\right) / w^{2} \\
& =\sum_{d \mid q} \frac{\mu^{2}(d)}{d} \sum_{\substack{w \leq \sqrt{x / d}, w \mid q^{\infty}}} \frac{\check{m}\left(x /\left(d w^{2}\right)\right)-1}{w^{2}}+\check{m}_{q}^{\sharp}(x)
\end{aligned}
$$

for a real parameter $U$ such that $x / q \geq X_{1}$,

$$
\begin{aligned}
\left|\check{m}_{q}(x)-\check{m}_{q}^{\sharp}(x)\right| & \leq \sum_{d \mid q} \frac{\mu^{2}(d)}{d} m^{*}(x / d) \\
& \leq \frac{q}{\varphi(q) \log (x / q)} \begin{cases}0.0538 & \text { when } x \geq 3158, \\
0.200 & \text { when } x \geq 101, \\
0.250 & \text { when } x>1 .\end{cases}
\end{aligned}
$$

\section{AN INTERMEDIATE FUNCTION FOR $\check{\check{m}}$}

In this part, we produce upper bounds for

$$
\check{\check{m}}^{*}(y)=\sum_{w \leq \sqrt{y+1}} \max _{y / w^{2} \leq z<(y+1) / w^{2}}\left|\check{m}(z)-2 \log z+2 \gamma_{0}\right| / w^{2}
$$

by following closely Section [10. We have again taken the maximum so that we may restrict our attention to integer values of $y$. With such a definition, our script will spend most of its time computing logarithms, which are costly. Let us investigate somewhat further what happens. The interval is $\left[y / w^{2},(y+1) / w^{2}\right)$. If there are no integers lying in this interval, then let us set

$$
A=\sum_{n \leq y / w^{2}} \frac{\mu(n)}{n}, B=\sum_{n \leq y / w^{2}} \frac{\mu(n) \log n}{n}, C=2 \gamma_{0}+\sum_{n \leq y / w^{2}} \frac{\mu(n)(\log n)^{2}}{n} .
$$

The function $\left|A(\log z)^{2}-2(B+1) \log z+C\right|$ is maximum at $\log z=\log \left(y / w^{2}\right)$, $\log z=\log \left((y+1) / w^{2}\right)$ or $\log z=(B+1) / A$ if this latter value falls within the proper bounds. If there is an integer, say $m$ lying in this interval, then we have to maximize $|A \log z-B|$ when $z \in\left[y / w^{2}, m\right)$ and a similar expression when $z \in\left[m, y /(w+1)^{2}\right)$. In both cases, we can recover the relevant $\log z$ in terms of the logarithms of integers $\leq y$. It is thus enough to precompute these.

Lemma 13.1. For integer $x$, we have

$$
\check{\check{m}}^{*}(x) \sqrt{x} \leq \begin{cases}0.333 & \text { when } x \in[16900,12000000] \\ 0.474 & \text { when } x \in[10,12000000] \\ 1.16 & \text { when } x \in[1,12000000]\end{cases}
$$


We precomputed the values of $\mu(n)$ and $\log n$ for $n$ up to $1.2 \cdot 10^{7}$, then the values of $m(x)$, of $-1+\sum_{n \leq x} \mu(n)(\log n) / n$ and of $2 \gamma_{0}+\sum_{n \leq x} \mu(n)(\log n)^{2} / n$ for $x$ up to $1.2 \cdot 10^{7}$. The total run time has been about 16 hours. The script is called AsymptoticBoundsFor_checkcheckmstar.gp and its main function is named getboundscheckcheckmstar.

We compared $\left|\check{m}^{*}(x)\right|$ with $1 / \sqrt{x}$ as this seems coherent with the numerical outputs. These outputs vary minimally, as shown by the following sample:

\begin{tabular}{cc||c} 
beginning & end & maximal constant \\
\hline 6800000 & 7000000 & $0.3318 \ldots$ \\
7000000 & 7200000 & $0.3313 \ldots$ \\
7200000 & 7400000 & $0.3318 \ldots$ \\
7400000 & 7600000 & $0.3318 \ldots$ \\
7600000 & 7800000 & $0.3316 \ldots$
\end{tabular}.

Question. Does there exist a constant $c$ such that $\lim _{x \rightarrow \infty} \sqrt{x}\left|\check{m}^{*}(x)\right|=c$ ?

We report also the results of a slight modification of the script used above.

Lemma 13.2. We have

$$
\left|\check{m}(x)-2 \log x+2 \gamma_{0}\right| \sqrt{x} \leq \begin{cases}0.00234 & \text { when } 3393 \leq x \leq 12000000, \\ 0.00650 & \text { when } 10 \leq x \leq 12000000, \\ 0.0717 & \text { when } 4 \leq x \leq 12000000 \\ 1.155 & \text { when } 1 \leq x \leq 12000000 .\end{cases}
$$

Lemma 13.3. We have

$$
\left|\check{m}^{*}(x)\right| \log x \leq \begin{cases}0.0408 & \text { when } x \geq 5 \\ 0.206 & \text { when } x>1 .\end{cases}
$$

Proof. The proof follows closely that of Lemma 10.4. but relies on Theorem 1.8 instead of Theorem 1.5, namely:

$$
\left|\check{m}(x)-2 \log x+2 \gamma_{0}\right| \leq 1 /(103 \log x) \quad(x \geq 9) .
$$

We also have $0 \leq \check{m}(x)-2 \log x+2 \gamma_{0} \leq 2 \gamma_{0}$ when $x \leq Y_{0}=16$, in agreement to the conjecture. Thus, for $x / U^{2}=Y_{0}$,

$$
\left|\check{m}^{*}(x)\right| \leq \frac{1}{103} \sum_{u \leq U} \frac{1}{u^{2} \log \left(x / u^{2}\right)}+2 \gamma_{0} \frac{1+U^{-1}}{U} .
$$

We continue by using a comparison with an integral

$$
\begin{aligned}
\left|\check{m}^{*}(x)\right| & \leq \frac{1}{103} \sum_{u \leq U} \frac{1}{u^{2} \log \left(x / u^{2}\right)}+2 \gamma_{0} \frac{1+U^{-1}}{U} \\
& \leq \frac{1}{103 \log x}+\frac{1}{103 \sqrt{x}} \int_{\sqrt{x / U^{2}}}^{\sqrt{x}} \frac{d v}{2 \log v}+2 \gamma_{0} \frac{1+U^{-1}}{U} \\
& \leq \frac{1}{103 \log x}+\frac{1}{103 \sqrt{x}} \int_{\sqrt{Y_{0}}}^{\sqrt{x}} \frac{d v}{2 \log v}+2 \gamma_{0} \frac{1+\sqrt{Y_{0} / x}}{\sqrt{x / Y_{0}}}
\end{aligned}
$$


We again employ Lemma 10.3 at this level. Hence, when $x \geq 1.2 \cdot 10^{7}$,

$$
\left|\check{\check{m}}^{*}(x)\right| \leq \frac{1}{103 \log x}+\frac{1.254}{103 \log x}+2 \gamma_{0} \frac{1+\sqrt{Y_{0} / x}}{\sqrt{x / Y_{0}}} \leq \frac{0.0408}{\log x} .
$$

We extend it to $x \geq 10$ by using Lemma 13.1, and to $x \geq 5$ by direct check, by modifying AsymptoticBoundsFor_checkcheckmstar.gp. We also have

$$
\left|\check{m}^{*}(x)\right| \leq 0.207 / \log x \quad(x>1) .
$$

\section{AN INTERMEDIATE FUNCTION FOR $\check{m}$, BIS REPETITA}

The previous section was dedicated to getting bounds of the shape $1 / \log x$ and we aim here at bounds of the shape $1 /(1+\log x)$. There are no difficulties, but the computations need to be put down.

Lemma 14.1. We have, for real $x$,

$$
\left|\check{\check{m}}^{*}(x)\right|(1+\log x) \leq \begin{cases}0.0452 & \text { when } x \geq 6 \\ 0.172 & \text { when } x \geq 2 \\ 1.155 & \text { when } x>1\end{cases}
$$

Proof. We follow the proof of Lemma 13.3 until (13.3) which we modify via Lemma 11.1 into

$$
\left|\check{\check{m}}^{*}(x)\right| \leq \frac{1}{103 \log x}+\frac{1.530}{103(1+\log x)}+2 \gamma_{0} \frac{1+\sqrt{Y_{0} / x}}{\sqrt{x / Y_{0}}} \leq \frac{0.0452}{1+\log x} .
$$

We extend it to $x \geq 540$ by using Lemma 7.1. We reduce this bound by direct verification, again by modifying AsymptoticBoundsFor_checkcheckmstar.gp .

\section{Proof of Theorems 1.16 and 1.17}

We proceed to prove the estimate concerning $\check{\check{m}}_{q}(x)$. Lemma 1.9 gives us,

$$
\begin{aligned}
\check{m}_{q}(x) & =\sum_{d \mid q} \frac{\mu^{2}(d)}{d} \sum_{\substack{w \leq \sqrt{x / d}, w \mid q^{\infty}}} \check{\check{m}}\left(x /\left(d w^{2}\right)\right) / w^{2} \\
& =\sum_{d \mid q} \frac{\mu^{2}(d)}{d} \sum_{\substack{w \leq \sqrt{x / d}, w \mid q^{\infty}}} \frac{\check{m}\left(x /\left(d w^{2}\right)\right)-2 \log \left(x /\left(d w^{2}\right)+2 \gamma_{0}\right.}{w^{2}}+\check{m}_{q}^{\sharp}(x) .
\end{aligned}
$$

Then, for a real parameter $U$ such that $x / q \geq X_{1}$, we have

$$
\left|\check{\check{m}}_{q}(x)-\check{\check{m}}_{q}^{\sharp}(x)\right| \leq \sum_{d \mid q} \frac{\mu^{2}(d)}{d} m^{*}(x / d) \leq \frac{q / \varphi(q)}{\log (x / q)} \begin{cases}0.0408 & \text { when } x \geq 5, \\ 0.206 & \text { when } x>1 .\end{cases}
$$




\section{Proof of Theorems 1.18 and 1.19}

We prove in this section estimates relative to $M_{q}$. As in the case of $m_{q}, \check{m}_{q}$ and $\check{\check{m}}_{q}$, we rely on an identity that links this quantity with its counterpart with no coprimality condition. Indeed, we readily modify the proof of Lemma 1.9 to get

$$
M_{q}(x)=\sum_{d \mid q} \mu^{2}(d) \sum_{\substack{w \leq \sqrt{x / d}, w \mid q^{\infty}}} M\left(\frac{x}{d w^{2}}\right) .
$$

We recall [20, Theorem 1]:

Lemma 16.1. For $D \geq 1078853$, we have

$$
\left|\sum_{d \leq D} \mu(d)\right| \leq \frac{0.0130 \log D-0.118}{(\log D)^{2}} D .
$$

We extend this bound to get the simpler

$$
\begin{aligned}
& \left|\sum_{d \leq D} \mu(d)\right| / D \leq \frac{0.0130}{\log D} \quad\left(D \geq D_{1}=97067\right), \\
& \left|\sum_{d \leq D} \mu(d)\right| / D \leq \frac{0.0950}{\log D} \quad\left(D \geq D_{0}=688\right) . \\
& \left|\sum_{d \leq D} \mu(d)\right| / D \leq \frac{0.644}{\log D} \quad(D>1) .
\end{aligned}
$$

The script we used is called AsymptoticBoundsFor_M.gp and its main function is getboundsM. But as in the previous cases of $m_{q}, \check{m}_{q}$ and $\check{m}_{q}$, simply plugging these estimates into (16.1) and estimating the tail via Rankin's trick leads to bad numerical results. We again take another path that requires many more computations but is numerically much better. We define

$$
M^{*}(y)=\sum_{w \leq \sqrt{y}} \max _{y / w^{2}<z \leq(y+1) / w^{2}}|M(z)| .
$$

Lemma 16.2. For an $n$ integer satisfying $99000 \leq n \leq 80000000$, we have

$$
M^{*}(n) \leq 0.574 \sqrt{n(1+\log n)} .
$$

It is enough to replace the constant 0.574 by 0.595 to extend this result to $x \geq 9933$. Furthermore, for $n$ integer satisfying $1 \leq n \leq 80000000$, we have

$$
M^{*}(n) \leq 2.403 \sqrt{n} \text {. }
$$

As it turns out, the quantity $M^{*}(n) / \sqrt{n(1+\log n)}$ tends to oscillate slowly and dips somewhat when $x$ increases while the quantity $M^{*}(n) / \sqrt{n}$ tends to rise slowly, still in an oscillating manner of course. Since we have to precompute only $M(n)$ which happens to be an integer of type small, the requirement in memory space is much less acute, when compared with the other computations of this kind we had to carry up to now. This is why we have been able to increase significantly the upper bound for $n$. The running time is, however, much larger, as it increases quadratically: on the same machine as before, it took about three days. The script is called AsymptoticBoundsFor_Mstar.gp and its main function is getboundsMstar.

Yet another numerical application of Lemma 7.2 yields: 
Lemma 16.3. With $D_{1}=97067$, the function

$$
T(y): y \mapsto \frac{\log y}{y} \int_{\sqrt{D_{1}}}^{y} \frac{d v}{\log v}
$$

is increasing and then decreasing, reaching a maximum around 45443.09 with value $1.102836+\mathcal{O}^{*}\left(10^{-7}\right)$.

All this has prepared the ground for estimating $\left|M^{*}(x)\right| \log x$.

Lemma 16.4. We have

$$
\left|M^{*}(x)\right| \log x \leq \begin{cases}0.0918 & \text { when } x \geq 48513 \\ 0.199 & \text { when } x \geq 4536 \\ 0.429 & \text { when } x \geq 490 \\ 0.997 & \text { when } x>1\end{cases}
$$

Proof. The proof is patterned on the one of Lemma 10.4. We find that, for $x / U_{0}^{2}=$ $D_{0}=688$ and $x / U_{1}^{2}=D_{1}=97067$,

$$
\begin{aligned}
M^{*}(x) / x \leq & \sum_{w \leq U_{1}} \frac{0.0130}{w^{2} \log \left(x / w^{2}\right)}+\sum_{U_{1}<w \leq U_{0}} \frac{0.0950}{w^{2} \log \left(x / w^{2}\right)}+\frac{1+U_{0}^{-1}}{U_{0}} \\
\leq & \frac{0.0130}{\log x}+\frac{0.0130}{\sqrt{x}} \int_{\sqrt{D_{1}}}^{\sqrt{x}} \frac{d v}{2 \log v}+\frac{0.0950 \sqrt{D_{1}}}{\sqrt{x} \log D_{1}} \\
& +\frac{0.0950}{\sqrt{x}} \int_{\sqrt{D_{0}}}^{\sqrt{D_{1}}} \frac{d v}{2 \log v}+\frac{1+\sqrt{D_{0} / x}}{\sqrt{x / D_{0}}} \\
\leq & \frac{0.0130(1+1.102836)}{\log x}+\frac{2.578}{\sqrt{x}}+\frac{2.777}{\sqrt{x}}+\frac{1+\sqrt{D_{0} / x}}{\sqrt{x / D_{0}}} \leq \frac{0.0918}{\log x}
\end{aligned}
$$

when $x \geq 80000000$. We use Lemma 16.2 to extend this bound to $x \geq 61408$ and to $x \geq 48513$ by direct inspection; this is done by modifying suitably the script AsymptoticBoundsFor_Mstar.gp

We can thus write

$$
\left|M_{q}(x)\right| \leq \sum_{d \mid q} \mu^{2}(d) M^{*}\left(\frac{x}{d}\right)
$$

and conclude as before.

Variation. We start with an application of Lemma 8.1.

Lemma 16.5. With $D_{1}=97067$, the function

$$
T^{*}(y): y \mapsto \frac{1+\log y}{y} \int_{\sqrt{D_{1}}}^{y} \frac{d v}{\log v}
$$

is increasing and then decreasing, reaching a maximum around 22545.85 with value $1.209488+\mathcal{O}^{*}\left(10^{-6}\right)$.

Lemma 16.6. We have

$$
\left|M^{*}(x)\right|(1+\log x) \leq \begin{cases}0.0975 & \text { when } x \geq 48645 \\ 0.198 & \text { when } x \geq 7100 \\ 0.498 & \text { when } x \geq 490 \\ 1 & \text { when } x>1\end{cases}
$$


Proof. We adapt the proof of Lemma 16.4 and get

$$
\begin{aligned}
M^{*}(x) / x & \leq \frac{0.0130}{\log x}+\frac{1.210 \times 0.0130}{1+\log x}+\frac{2.578}{\sqrt{x}}+\frac{2.777}{\sqrt{x}}+\frac{1+\sqrt{D_{0} / x}}{\sqrt{x / D_{0}}} \\
& \leq \frac{0.0975}{1+\log x}
\end{aligned}
$$

when $x \geq 80000000$. We use Lemma 16.2 to extend this bound to $x \geq 66000$ and to $x \geq 48645$ by direct inspection, again by modifying suitably the script AsymptoticBoundsFor_Mstar.gp.

\section{ACKNOWLEDGEMENT}

The referee is thanked for his/her careful reading and constructive remarks that have helped to improve this paper.

\section{REFERENCES}

[1] A. Axer, Über einige Grenzwertsätze, Wien. Ber. 120 (1911), 1253-1298 (German).

[2] M. Balazard, Remarques élémentaires sur la fonction de Moebius, private communication (2011).

[3] — Elementary remarks on Möbius' function, Proceedings of the Steklov Intitute of Mathematics 276 (2012).

[4] Paul T. Bateman and Harold G. Diamond, Asymptotic distribution of Beurling's generalized prime numbers, Studies in Number Theory, Math. Assoc. Amer. (distributed by PrenticeHall, Englewood Cliffs, N.J.), 1969, pp. 152-210. MR.0242778 (39 \#4105)

[5] D. Berkane, O. Bordellès, and O. Ramaré, Explicit upper bounds for the remainder term in the divisor problem, Math. Comp. 81 (2012), no. 278, 1025-1051, DOI 10.1090/S0025-57182011-02535-4. MR2869048

[6] N. Costa Pereira, Elementary estimates for the Chebyshev function $\psi(x)$ and for the Möbius function $M(x)$, Acta Arith. 52 (1989), no. 4, 307-337. MR1030085 (91e:11099)

[7] H.G. Diamond and Wen-Bin Zhang, A PNT equivalence for Beurling numbers, Submitted to Functiones et approximatio (2012).

[8] François Dress and Mohamed El Marraki, Fonction sommatoire de la fonction de Möbius. II. Majorations asymptotiques élémentaires (French, with English and French summaries), Experiment. Math. 2 (1993), no. 2, 99-112. MR1259424 (95d:11178)

[9] M. El Marraki, Fonction sommatoire de la fonction de Möbius. III. Majorations asymptotiques effectives fortes (French, with English and French summaries), J. Théor. Nombres Bordeaux 7 (1995), no. 2, 407-433. MR1378588 (97e:11113)

[10] M. El Marraki, Majorations de la fonction sommatoire de la fonction $\frac{\mu(n)}{n}$, Univ. Bordeaux 1 Pré-publication (1996), no. 96-8.

[11] Andrew Granville and Olivier Ramaré, Explicit bounds on exponential sums and the scarcity of squarefree binomial coefficients, Mathematika 43 (1996), no. 1, 73-107, DOI 10.1112/S0025579300011608. MR:1401709 (97m:11023)

[12] Alfred Kienast, Über die Äquivalenz zweier Ergebnisse der analytischen Zahlentheorie (German), Math. Ann. 95 (1926), no. 1, 427-445, DOI 10.1007/BF01206619. MR1512286

[13] R. A. MacLeod, Errata to the paper "A new estimate for the sum $M(x)=\Sigma_{n \leq x} \mu(n)$ ", Acta Arith. 16 (1969/1970), 99-100. MR0245541 (39 \#6847)

[14] R. A. MacLeod, A curious identity for the Möbius function, Utilitas Math. 46 (1994), 91-95. MR 1301298 (95g:11002)

[15] H.L. Montgomery and R.C. Vaughan, Multiplicative Number Theory: I. Classical Theory, Cambridge Studies in Advanced Mathematics, vol. 97, Cambridge University Press, 2006.

[16] Yōichi Motohashi, Primes in arithmetic progressions, Invent. Math. 44 (1978), no. 2, 163178. MR0472739 (57 \#12429b)

[17] Nathan Ng, The distribution of the summatory function of the Möbius function, Proc. London Math. Soc. (3) 89 (2004), no. 2, 361-389, DOI 10.1112/S0024611504014741. MR2078705 (2005f:11215) 
[18] The PARI Group, Bordeaux, PARI/GP, version 2.5.2, 2011, http://pari.math. u-bordeaux.fr/.

[19] O. Ramaré, Explicit estimates on the summatory functions of the Moebius function with coprimality restrictions, Submitted (2013), 12pp.

[20] Olivier Ramaré, From explicit estimates for primes to explicit estimates for the Möbius function, Acta Arith. 157 (2013), no. 4, 365-379, DOI 10.4064/aa157-4-4. MR3019422

[21] Olivier Ramaré, Some elementary explicit bounds for two mollifications of the Moebius function, Functiones et Approximatio (2013), 12pp.

[22] Barkley Rosser, Explicit bounds for some functions of prime numbers, Amer. J. Math. 63 (1941), 211-232. MR0003018 (2,150e)

[23] J. Barkley Rosser and Lowell Schoenfeld, Approximate formulas for some functions of prime numbers, Illinois J. Math. 6 (1962), 64-94. MR0137689 (25 \#1139)

[24] Lowell Schoenfeld, An improved estimate for the summatory function of the Möbius function, Acta Arith. 15 (1968/1969), 221-233. MR0241376 (39 \#2716)

[25] Wolfgang Schwarz and Jürgen Spilker, Arithmetical Functions, An Introduction to Elementary and Analytic Properties of Arithmetic Functions and to Some of Their Almost-Periodic Properties. London Mathematical Society Lecture Note Series, vol. 184, Cambridge University Press, Cambridge, 1994. MR.1274248 (96b:11001)

Laboratoire CNRS Paul Painlevé, Université Lille 1, 59655 Villeneuve d'AscQ, France

E-mail address: ramare@math.univ-lille1.fr 